ARTICLE

\title{
Mechanism of lipid droplet formation by the yeast Sei1/Ldb16 Seipin complex
}

\author{
Yoel A. Klug (1) 1, Justin C. Deme1,2,5, Robin A. Corey 3,5, Mike F. Renne (i) 1,5, Phillip J. Stansfeld (1) 3,4,
} Susan M. Lea (1) ${ }^{1,2 \otimes} \&$ Pedro Carvalho (i) ${ }^{1 凶}$

Lipid droplets (LDs) are universal lipid storage organelles with a core of neutral lipids, such as triacylglycerols, surrounded by a phospholipid monolayer. This unique architecture is generated during LD biogenesis at endoplasmic reticulum (ER) sites marked by Seipin, a conserved membrane protein mutated in lipodystrophy. Here structural, biochemical and molecular dynamics simulation approaches reveal the mechanism of LD formation by the yeast Seipin Sei1 and its membrane partner Ldb16. We show that Sei1 luminal domain assembles a homooligomeric ring, which, in contrast to other Seipins, is unable to concentrate triacylglycerol. Instead, Sei1 positions Ldb16, which concentrates triacylglycerol within the Sei1 ring through critical hydroxyl residues. Triacylglycerol recruitment to the complex is further promoted by Sei1 transmembrane segments, which also control Ldb16 stability. Thus, we propose that LD assembly by the Sei1/Ldb16 complex, and likely other Seipins, requires sequential triacylglycerol-concentrating steps via distinct elements in the ER membrane and lumen.

\footnotetext{
${ }^{1}$ Sir William Dunn School of Pathology, University of Oxford, Oxford, UK. ${ }^{2}$ Center for Structural Biology, Center for Cancer Research, National Cancer Institute, Frederick, MD, USA. ${ }^{3}$ Department of Biochemistry, University of Oxford, Oxford, UK. ${ }^{4}$ School of Life Sciences \& Department of Chemistry, University of Warwick, Coventry, UK. ${ }^{5}$ These authors contributed equally: Justin C. Deme, Robin A. Corey, Mike F. Renne. ${ }^{\varpi_{e}}{ }$ ail: susan.lea@nih.gov; pedro.carvalho@path.ox.ac.uk
} 
ipid droplets (LDs) are storage organelles for neutral lipids, such as triacylglycerol (TAG), with central roles in lipid and energy metabolism ${ }^{1,2}$. Despite their importance and links to metabolic diseases ${ }^{3}$, the mechanisms controlling LD formation and dynamics remain poorly understood.

LDs display a unique architecture with a hydrophobic core composed of neutral lipids enclosed in a phospholipid monolayer ${ }^{4,5}$ with a set of LD-specific proteins ${ }^{6,7}$. In recent years, various proteins and lipids have been implicated in LD biogenesis, and a general model of LD biogenesis has emerged ${ }^{8,9}$. Assembly of LDs occurs at the endoplasmic reticulum (ER) ${ }^{10,11}$, and is dependent on synthesis of neutral lipids ${ }^{12}$. At low concentrations neutral lipids disperse within the acyl chain region of the ER membrane lipids, but as their concentration rises, they phase separate from surrounding phospholipids and coalesce into a lens-like structure between the leaflets of the ER bilayer ${ }^{13-15}$. As this neutral lipid lens grows, it gives rise to a nascent LD that buds from the ER enclosed in a phospholipid monolayer derived from the cytosolic leaflet of the ER bilayer ${ }^{16}$.

The ER sites of LD biogenesis are marked by Seipin, an evolutionarily conserved ER membrane protein ${ }^{17-21}$. In humans, Seipin is encoded by the Bernadelli-Seip congenital lipodystrophy type 2 (BSCL2), a gene frequently mutated in familial forms of lipodystrophy ${ }^{22}$. At the cellular level, loss of Seipin results in aberrant LDs, which become tiny, often clustered, interspersed with a few supersized $\mathrm{LDs}^{21,23,24}$, likely due to impaired maturation ${ }^{17}$ and contacts with the $\mathrm{ER}^{19,20}$. Acute depletion of Seipin in human cells resulted in heterogenous LDs, demonstrating that Seipin is also required for the maintenance of mature $\mathrm{LDs}^{25}$.

Seipin has two transmembrane (TM) segments proximal to the $\mathrm{N}$ - and C-termini separated by an extended luminal domain ${ }^{26}$. Cryogenic-electron microscopy (cryo-EM) structures of human and fly Seipin luminal domains showed homo-oligomeric ring assemblies of 11 and 12 subunits, respectively, an arrangement essential for function ${ }^{27,28}$. Despite the different subunit number within the Seipin rings, the individual Seipin protomers adopted a similar fold with 8 -strand $\beta$-sandwich typical of certain lipidbinding proteins. While capable of binding anionic phospholipids in vitro, the in vivo relevance of these observations is still unclear $^{27}$. Both in human and fly Seipin, the luminal $\beta$-sandwich is capped by a hydrophobic helix, which in the oligomer is positioned at the inner surface of the ring, protruding into the luminal leaflet of the ER bilayer. Recent molecular dynamics (MD) simulations showed that key serine residues in the hydrophobic helix interact directly with TAG within the membrane. Given its position at the center of the Seipin ring, this hydrophobic helix effectively concentrates TAG molecules, thereby facilitating lens formation and LD budding 29,30 . This hydrophobic helix was also shown to bind to Promethin/ LDAF1 $1^{30,31}$, a conserved ER membrane protein homologous to yeast $\operatorname{Ldo} 45^{32-34}$.

In contrast to mammalian and fly Seipins, the function of yeast Seipin Sei1 requires the ER membrane protein Ldb16, and LD defects observed in sei1,$l d b 16 \Delta$ and sei1 $\Delta l d b 16 \Delta$ cells are indistinguishable ${ }^{18,35}$. Curiously, expression of human Seipin in

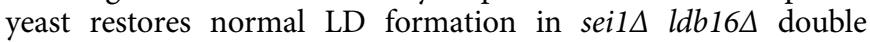
mutants ${ }^{18}$, indicating that in yeast, Seipin function is distributed between two polypeptides.

Here, we explored this unique organization of yeast Seipin to define the mechanism of Seipin-mediated LD formation. We show that Seil forms a homodecameric ring that scaffolds and positions Ldb16, with the latter emerging as the primary TAG binder via specific hydroxyl-containing residues, as proposed for human Seipin. Our structure revealed a structural element, the locking helix, which enables a distinctive arrangement of Sei1 TM segments, critical for both Ldb16 stability and the initial recruitment of TAG to the Sei1/Ldb16 complex. We propose that the dual TAG interaction through the TM segments and central ring elements define a unifying mechanism for Seipin-mediated LD formation and lipid storage.

\section{Results}

Cryo-electron microscopy structure of yeast Sei1. To gain insight into the mechanism of LD assembly by Seipin we determined the structure of the yeast Seipin Seil. A functional C-terminally 3xFLAG tagged Seil (Sei1-FLAG) was overexpressed using a galactose-inducible promoter in S. cerevisiae. Seil-FLAG was affinity purified from crude membranes solubilized in dodecyl maltoside (DDM), and supplemented with cholesterol hemisuccinate (CHS), followed by size-exclusion chromatography. Sei1-FLAG eluted in a single high molecular weight peak (Fig. S1A and B), which exhibited large homogeneous particles when analyzed by negative stain EM (Fig. S1C) suggesting it to be an oligomer. Next, the peak fraction of Seil-FLAG was analyzed by single particle cryo-EM (Fig. 1A and S1D-F). The structure of Sei1 was determined to a resolution of $2.7 \AA$ (Fig. 1A, B and S1D-F). The Seipin ring is assembled from 10 Seil protomers with a diameter of $140 \AA$ in the outer and $25 \AA$ in the inner rings (Fig. 1A, B). The Seil decamer contrasted with the undecameric and dodecameric rings assembled by human ${ }^{27}$ and $\mathrm{fly}^{28}$ Seipins, respectively. Seil protomers are characterized by an 8 -strand $\beta$ sandwich capped by two orthogonal short helices ( $\alpha 1$ and $\alpha 2)$ at the inner surface of the ring protruding slightly towards the membrane side (Fig. 1C). The Seil $\beta$-sandwich fold (Fig. 1C) is reminiscent of the one present in the cholesterol binding $\mathrm{NPC}^{36}$ (Fig. S1G), as observed for the $\beta$-sandwiches in human ${ }^{27}$ and fly ${ }^{28}$ Seipin luminal domains (Fig. 1D). Despite the differences in subunit number, a ring-like arrangement composed of $\beta$-sandwich motifs appears to be a general feature for Seipin function across eukaryotes.

Besides the luminal domain and in contrast to previous studies, our Seil structure also revealed regions proximal to and within the membrane. On the outer diameter of the Seil ring, just underneath the $\beta$-sandwich, we observed a short helix parallel to the plane of the membrane ( $\alpha 3$ ) that sits on top of the two TM segments (Fig. 1C). Although the two TM segments are encoded by amino acids at the very $\mathrm{N}$ - and $\mathrm{C}$-terminal of Seil sequence, they come into close proximity and show extensive interactions in the folded polypeptide (Fig. 1C).

The Seil luminal domain lacks a hydrophobic helix and the ability to concentrate TAG. A luminal helical region on the inner surface of the Seipin ring $(\alpha 1, \alpha 2)$, projecting towards the ER membrane (Figs. 1D and 2A), is present across eukaryotes. However, we observed marked differences in the size, position, and properties of the Seil helical region when compared to the equivalent portion of human and fly Seipins. In human and fly Seipins the helix is large, slightly kinked (Fig. 2A), and combines hydrophobic (Fig. 2A) and uncharged (Fig. S2A) amino acids, hence it has been referred to as hydrophobic helix ${ }^{27,28}$. Due to its size and amino acid composition, the hydrophobic helix of human and fly Seipin insert in the luminal leaflet of the bilayer (Fig. 2B). Importantly, recent MD simulations indicate a functional role of the hydrophobic helix within the membrane through conserved serine residues that interact directly with TAG carbonyl groups, resulting in effective concentration of TAG within the Seipin rings ${ }^{29,30}$. In contrast, the helical region in yeast 
A

(i)

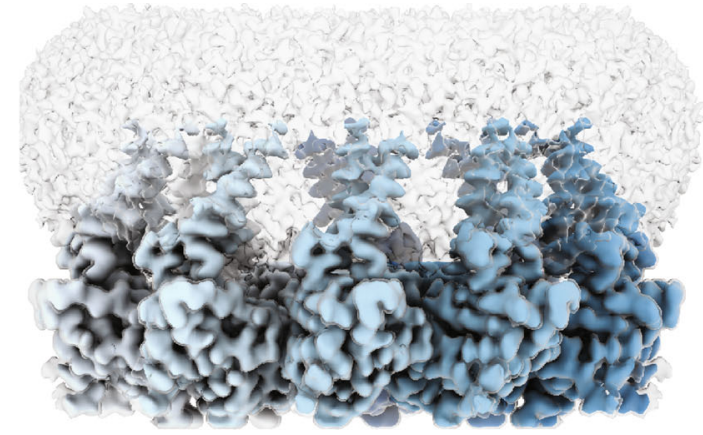

(ii)

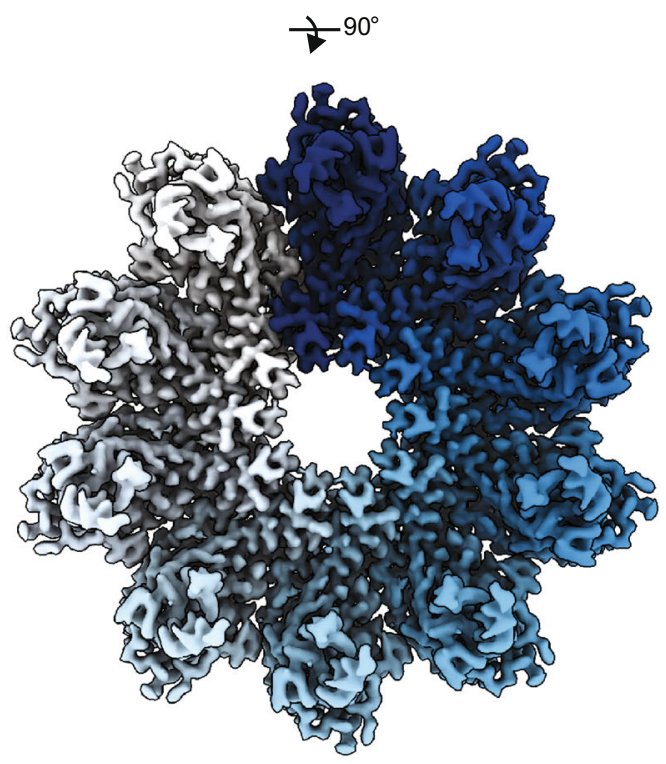

C
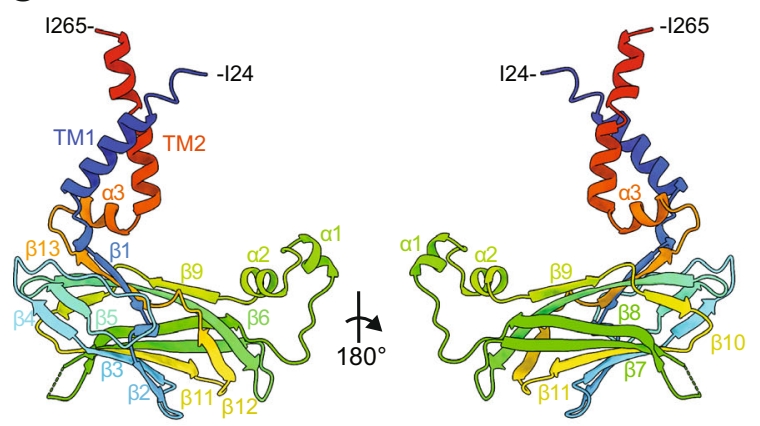

B

(i)

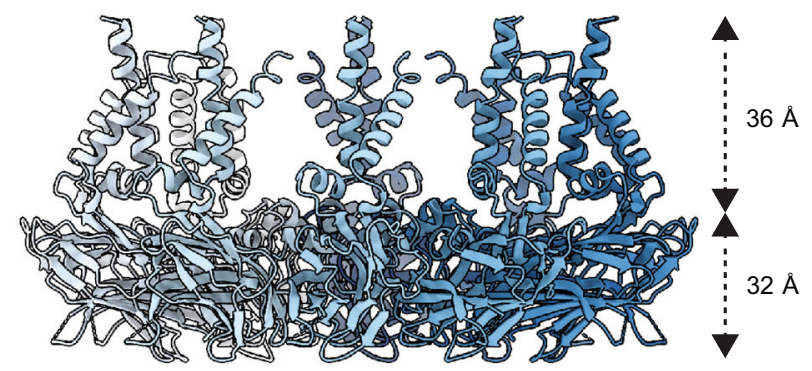

(ii)

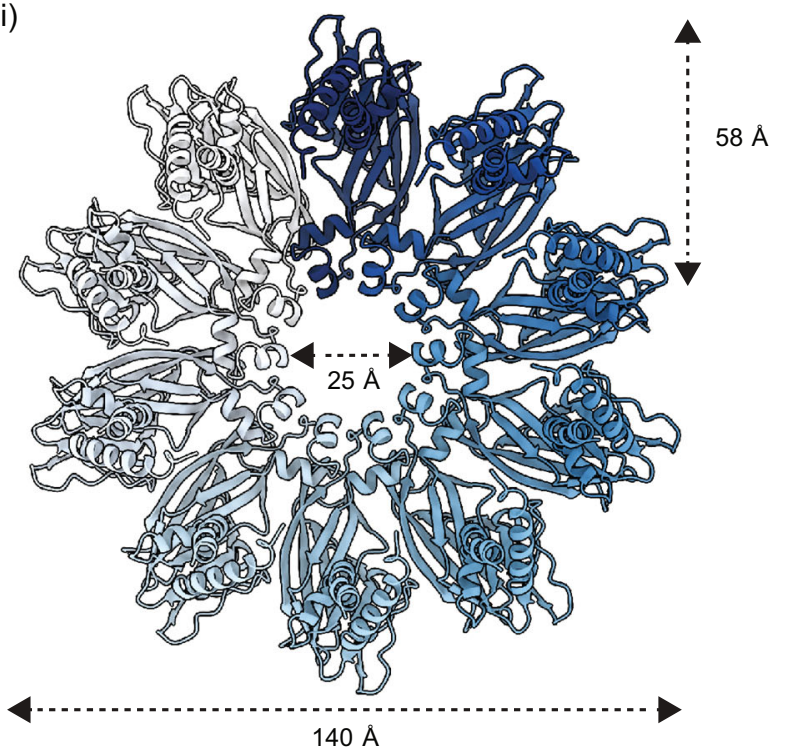

D

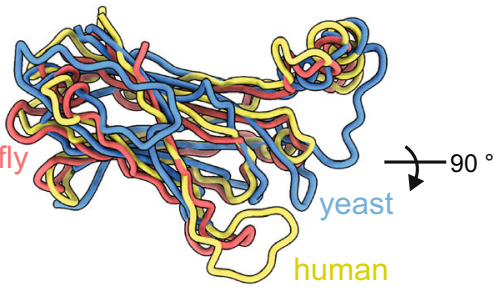

rmsd $3.26 \AA$

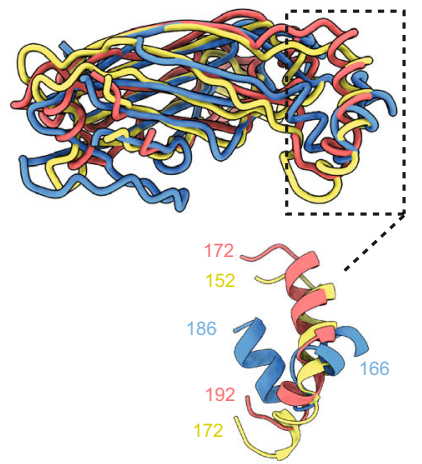

Fig. 1 Structure of the yeast Seipin Sei1. A Cryo-EM map of Sei1 homodecamer shown as (i) side view, depicting protein density (contour level of 0.007) with each Sei1 protomer colored in shades of blue, and the surrounding detergent micelle shown in transparent gray (contour level of 0.005) or (ii) 90degree rotated view looking down from the cytosol, with detergent micelle omitted for clarity (contour level of 0.007). B Cartoon representation of the Sei1 homodecamer model depicted as (i) side view or (ii) 90-degree rotated view looking down from the cytosol, as depicted in (A). Individual Sei1 protomers are displayed in different shades of blue. C Cartoon of Sei1 protomer model colored as rainbow from N- (blue) to C-(red) termini). The various Sei1 domains are indicated: partial transmembrane helices (TM1 and TM2) capped by the short $\alpha 3$ helix; the luminal $\beta$-sandwich ( $\beta$-strands 1-13); and central helices $\alpha 1$ 2. D Structural alignment of the luminal domains of yeast (blue; residues 49-232), fly (residues 88-240 of PDB 6MLU; orange), and human (residues 60-219 of PDB 6DS5; yellow) Seipin protomers. Indicated RMSD is against the yeast protomer. Inset-detail of the luminal helices protruding towards the ER membrane. 

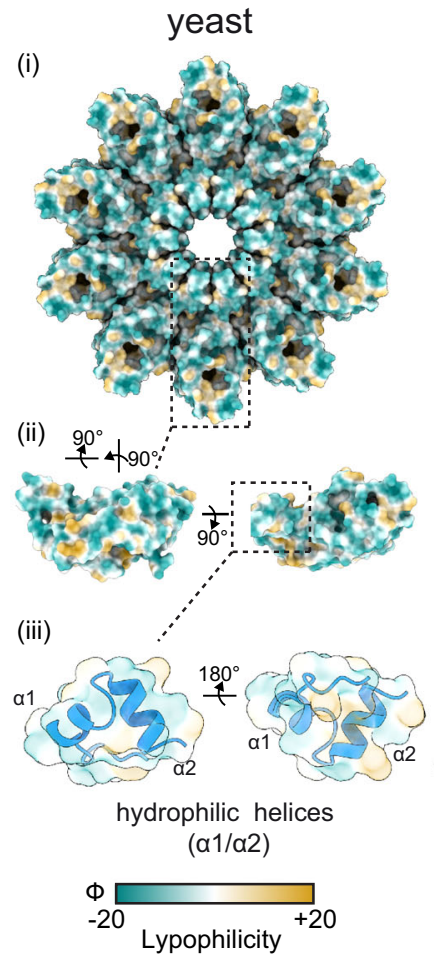

(iii)

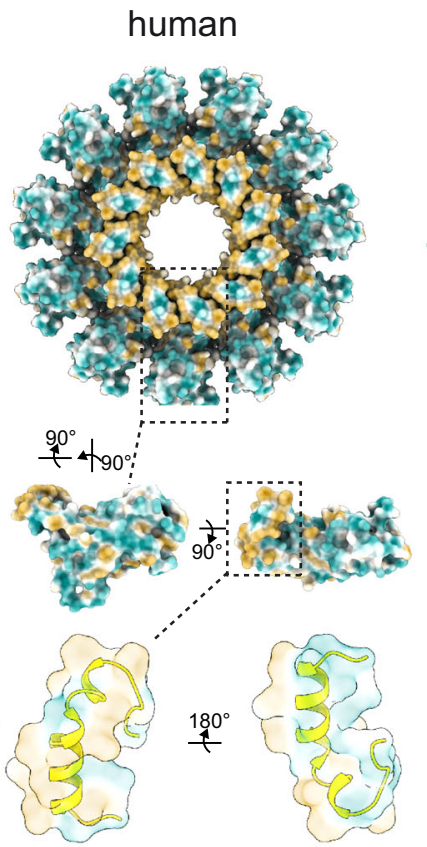

hydrophobic helix
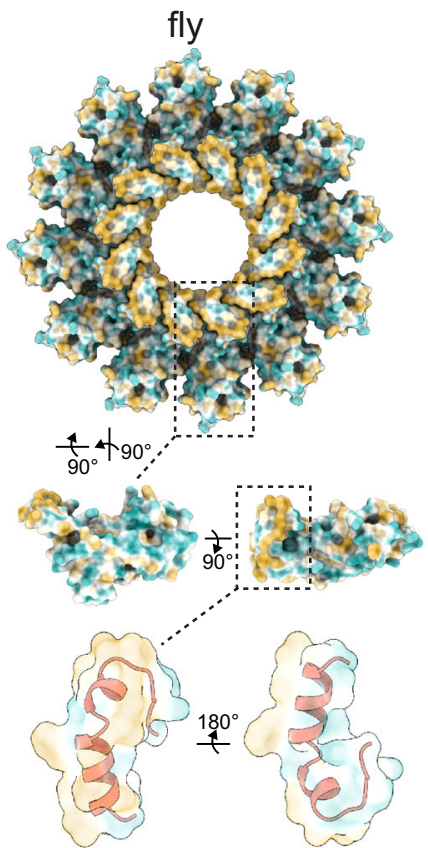

hydrophobic helix

B

yeast

human

fly
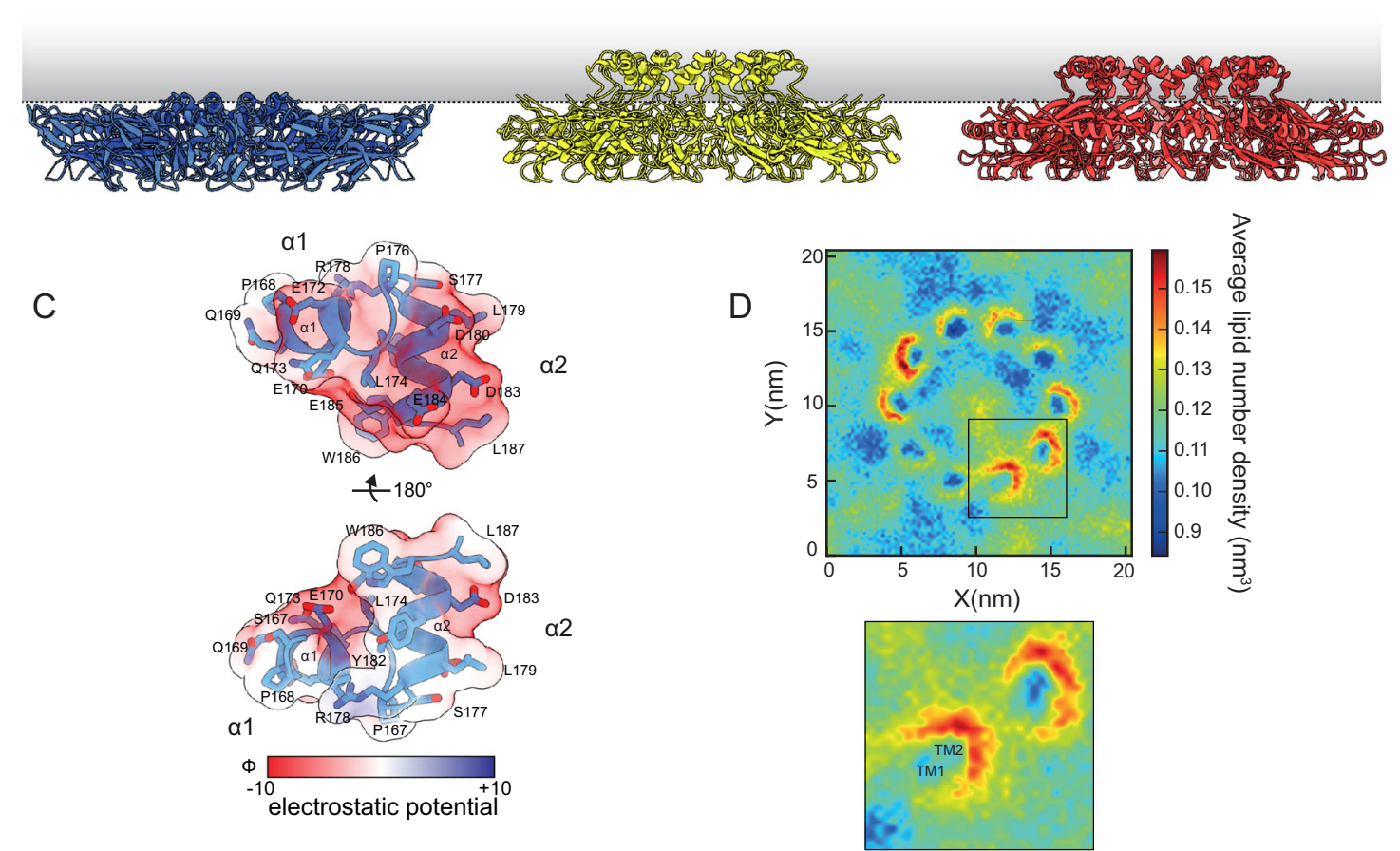

Fig. 2 The Sei1 luminal domain lacks a hydrophobic central helix and the ability to concentrate TAG. A Lipophilicity potential mapped to surface representations of yeast (left), human (middle), and fly (right) as viewed as (i) homodecamer assembly from the cytosol, (ii) individual protomers, or (iii) transparent overlay over zoomed in cartoon representation of the central $\alpha 1-\alpha 2$ helices. Surfaces are colored from hydrophilic (dark cyan) to hydrophobic (gold). Lipophilicity potential was generated from the mlp command of ChimeraX. B Side view of the luminal domains of yeast, human (PDB 6DS5) and fly (PDB 6MLU) Seipin in relation to the plane of the ER membrane (indicated by a dotted line). C Charge distribution of the yeast Sei1 central helices ( $\alpha 1, \alpha 2$ ), depicted as a transparent Coulombic electrostatic potential surface representation (Red, negative charge; blue, positive charge; white, no charge) overlayed over a cartoon representation (light blue) to show acidic side chains. Electrostatic potential was generated from the coulombic command of ChimeraX. D Top View of coarse-grained MD simulations of Sei1 in a POPC membrane with 3\% trioleylglycerol. Images depict average lipid number density of trioleylglycerol. Inset - zoom in of corresponding box showing positions of TM1 and TM2. 
Sei1 luminal domain is split into two orthogonal small helices ( $\alpha 1$ and a2) (Fig. 2A), and does not extend into the membrane (Fig. 2B). Furthermore, the Seil a1/ a2 region is enriched in charged residues (Fig. 2C, Fig. S2A). Coarse-grained MD simulations showed that TAG fails to concentrate within the rings of yeast Seil, consistent with it lacking a membrane protruding hydrophobic helix (Fig. 2D). Curiously, mild but consistent TAG enrichment was observed in proximity of Seil TM segments suggesting a contribution of the membrane region in LD formation (Fig. 2D). In contrast, similar simulations using the human Seipin luminal domain showed dramatic TAG accumulation within the ring (Fig. S2B), as previously described ${ }^{29,30}$. Thus, the lack of membrane embedded hydrophobic helix indicates that yeast Seil uses an alternative mechanism for concentrating TAG during LD formation.

Ldb16 complements Seil structure for LD formation. In contrast to human and fly Seipin, Seil-mediated LD formation depends on Ldb16, a yeast specific binding partner. This raises the possibility that Ldb16 complements the Seil structure by providing the hydrophobic helix required for TAG concentration within the ring. Consistent with this possibility, expression of human Seipin restores normal LD formation in yeast mutants lacking both Seil and $\mathrm{Ldb} 16^{18}$. We attempted to explore this hypothesis using a structural approach on purified Seil/Ldb16 complex. A functional fusion of Ldb16 to streptavidin binding protein (Ldb16-SBP) and Seil-FLAG were co-overexpressed, and the complex was purified as described above. Although Sei1FLAG and Ldb16-SBP co-purified (Fig. S3A, B), the EM map obtained with Sei1/Ldb16 complex was indistinguishable from the one obtained with Seil alone suggesting that Ldb16 was lost during sample vitrification.

To determine the positioning of Ldb16 in relation to the Sei1 ring we instead employed in vivo site-specific photo-crosslinking. A phenylalanine derivative carrying a photoreactive benzophenone (Bpa) was incorporated in Seil-FLAG at positions specified by an amber stop codon, as described ${ }^{37}$. The photoreactive probe was individually placed at several positions within the first and second TM segments (TM1 and TM2, respectively) as well as in the luminal domain, including the two short hydrophilic $a 1 / \alpha 2$ helices unique to Seil. Cells expressing Seil-FLAG with individual Bpa probes and endogenous Ldb16 tagged with HA (Ldb16-HA) were UV-irradiated to trigger protein crosslinking. Strong ladder-like Seil-Seil crosslinks were observed for probes inserted in the luminal region but not when Bpa was within TMs (Fig. 3A). This is in agreement with our structural data showing that interactions between Seil protomers occurs through the luminal domain. Prominent Seil-Ldb16 crosslinks were also detected for specific Bpa probes in TM1, TM2 and central luminal region confirming an intimate relationship between the two proteins (Fig. 3A, B). Remarkably, the Seil a1/a2 helices showed strong crosslinks to Ldb16, particularly in residues pointing towards the center of the Seil ring. This indicates that Ldb16 is well-positioned to concentrate TAG. To test the importance of Sei1 a1/a2 helices in controlling Ldb16 positioning and LD formation we replaced this Seil region by a flexible linker (Sei1GGSGGS) (Fig. S3C). This mutant was expressed to levels comparable to WT Seil (Fig. S3D) and oligomerized efficiently (Fig. S3E). Ldb16 levels were not affected (Fig. S3D) and it coprecipitated with the mutant Seil GGSGGS similarly as with WT (Fig. S3F), suggesting the formation of a stable complex. However, cells expressing Sei1 GGSGGS had mildly enlarged LDs (Fig. 3C, D). Using a well-established assay to monitor de novo LD formation upon induction of a single TAG biosynthetic gene $^{10}$, we observed that cells expressing Seil ${ }^{\mathrm{GGSGGS}}$ were also defective in LD biogenesis, with a reduced number of LDs forming over time (Fig. S3G, H). Thus, Sei1 a1/a2 helices interact with Ldb16 possibly facilitating its positioning for TAG concentration during $\mathrm{LD}$ formation.

Ldb16 has $\mathrm{N}$ - and C-termini in the cytosol ${ }^{18}$, and is predicted to have two TMs. In silico structure prediction by trRosetta ${ }^{38}$ revealed an Ldb16 element with remarkable similarities to the TAG concentrating helices in human and fly Seipins (Fig. 3E). In between the two Ldb16 TM segments, there is a short helical region rich in serine (S53, S55, S62) and threonine (T52, T61, T63) residues, which through their hydroxyl groups could potentially be involved in TAG binding, as observed for human and fly Seipins.

To test the functional relevance of these hydroxyl-rich motifs, we generated several mutants in the S/T-residues. These mutants were expressed to normal levels (Fig. S3I) and bound endogenous Seil (Fig. S3J). Remarkably, these Ldb16 mutants displayed aberrant LDs (Fig. 3F, G) consistent with a role of the hydroxyl-containing residues in TAG concentration. Altogether, these data strongly support a model in which Seil ring extensively interacts with Ldb16 facilitating its positioning for TAG concentration through a mechanism analogous to human and fly Seipins.

The Seil Locking Helix positions transmembrane segments and facilitates Ldb16 binding. Ldb16 protein levels and stability depend on Sei1 ${ }^{18}$. While our photo-crosslinking approach shows extensive interaction between the two proteins, deletion of Sei1 a1/a2 helices did not affect Ldb16 levels (Fig. S3D). This observation suggested that interactions with the TM regions are important for Ldb16 stability. To test this possibility various residues within Seil TM1 and TM2 were mutated. When expressed from the endogenous Seil promoter all these mutants displayed low steady state levels (Fig. S4A). Consequently, Ldb16 levels were also reduced (Fig. S4A) and LDs were aberrant (Fig. S4B). However, if expressed from the alcohol dehydrogenase promoter $(A D H 1 \mathrm{p})$, a strong constitutive promoter, all Sei1 TM mutants showed levels comparable to WT Seil (Fig. $\mathrm{S} 4 \mathrm{C}$ ). Importantly, for most mutants this also resulted in the restoration of Ldb16 levels (Fig. S4C) and normal LDs (Fig. S4B).

The notable exception was the mutation of tyrosine residues at positions 37 and 41 (Y37 and Y41, respectively). Proximal to the luminal face of the membrane, Y37/Y41 establish methionine-aromatic interactions with a methionine residue (M240) in a short helix ( $\alpha 3)$ in between the luminal $\beta$-sandwich and the TM region, hereafter called locking helix (LH) (Fig. 4A). In cells expressing Sei1 $1^{\mathrm{LL}}$, a mutant where the aromatic residues (Y37/Y41) were replaced by leucines (L), Ldb16 levels remained low (Fig. S4C). Similarly, cells expressing Sei1 $\Delta \mathrm{LH}$, where a flexible linker replaced the LH (Fig. S4D), also failed to stabilize Ldb16 (Fig. S4C). Moreover, Sei1 ${ }^{\Delta \mathrm{LH}}$ and Sei1 ${ }^{\mathrm{LL}}$ cells displayed aberrant LDs however, in comparison to seils mutant, the frequency of supersized LDs was reduced (Fig. 4B, C). Thus, disruption of the methionine-aromatic interactions by mutating the $\mathrm{LH}$ or key aromatic residues in TM1 results in LD morphology defects.

To gain further insight of how methionine-aromatic interactions influenced the behavior of Seil TM segments we employed MD. Atomistic simulations reveal that in WT Seil, TM1 and TM2 adopt a stable conformation and maintain a constant angle (Fig. 4D) indicating that they move largely as a single unit (Fig. 4E). This coupling of Seil TM movement is impaired upon 

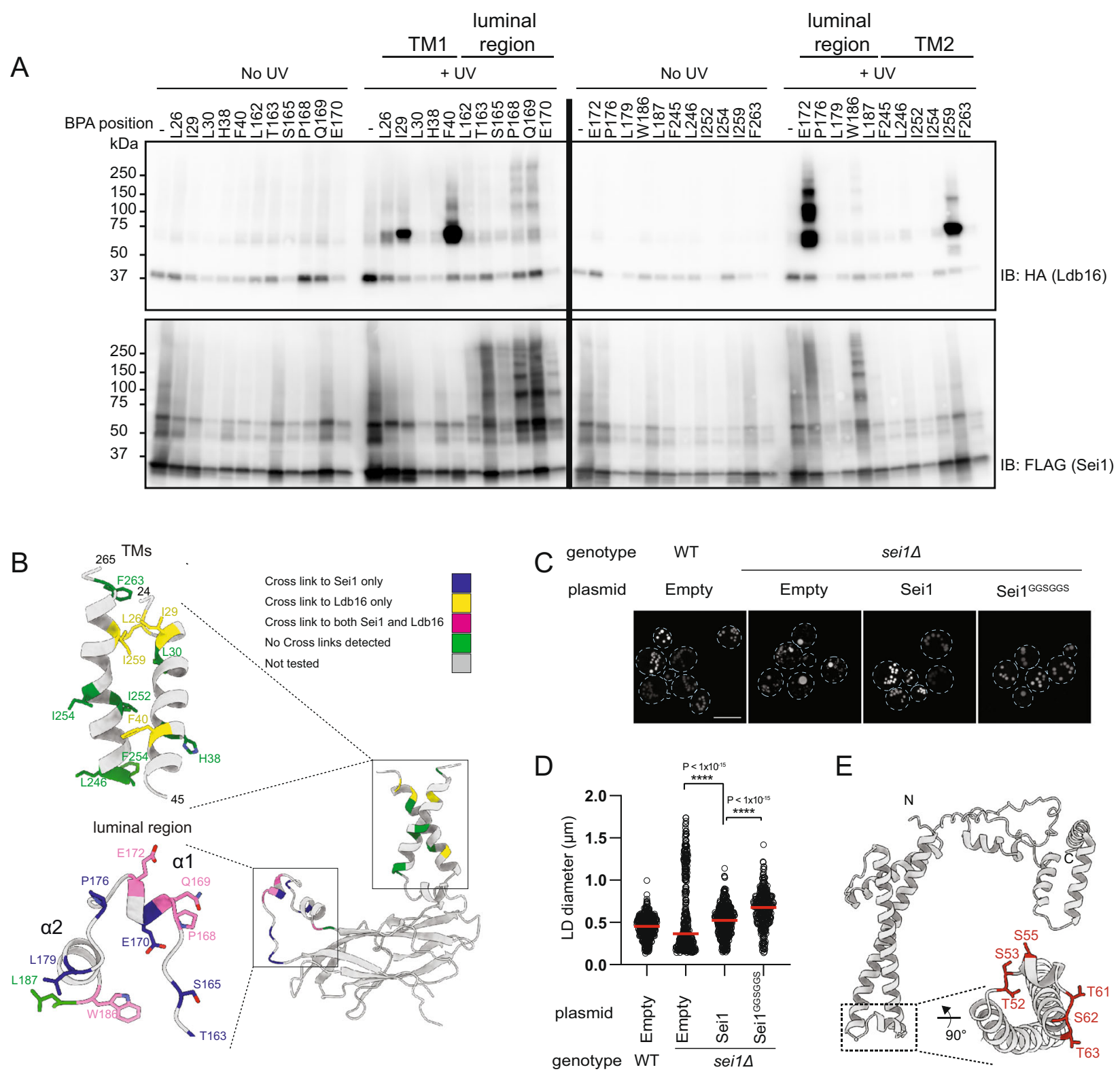

$E$

$\mathrm{F}$

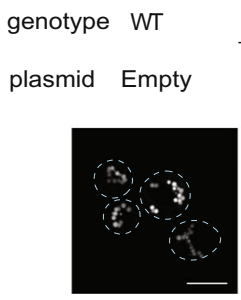

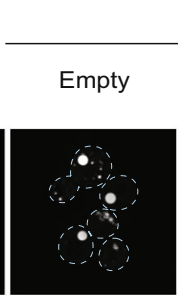

$I d b 16 \Delta$

Ldb16 3A

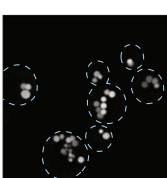

\section{Ldb16 6A}

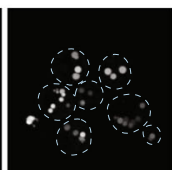

Ldb16 (44-59 polyAAS

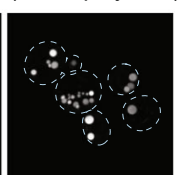

G

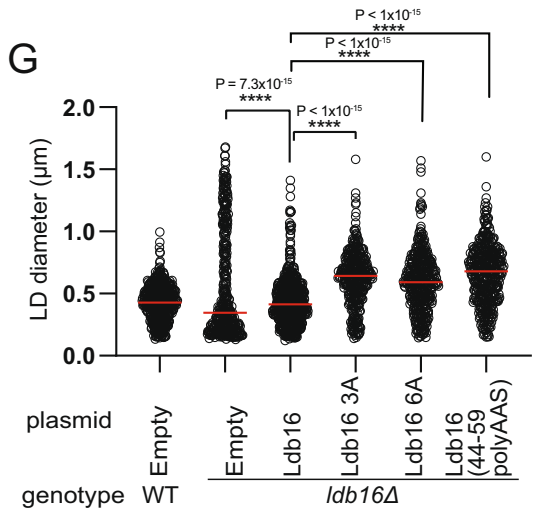

disruption of the methionine-aromatic interactions, in Sei1 ${ }^{\Delta \mathrm{LH}}$ and Sei1 ${ }^{\mathrm{LL}}$ (Fig. 4E). Besides their uncoordinated movement, $\mathrm{TM}$ segments in Sei1 ${ }^{\Delta \mathrm{LH}}$ and Sei1 ${ }^{\mathrm{LL}}$ appear to explore a higher membrane area. Importantly, the relative position of the TM domains to the Seil luminal domain did not appear to be affected by the mutations (Fig. S4E). To further demonstrate that the effect of methionine-aromatic interactions was restricted to the positioning and dynamics of the TM segments we solved the structure of Sei1 ${ }^{\Delta \mathrm{LH}}$. FLAG-tagged Sei1 ${ }^{\mathrm{LL}}$ was expressed and purified as above (Fig. S4F and G), and analyzed 
Fig. 3 Ldb16 complements Sei1 structure for LD formation. A sei1 cells expressing endogenously HA-tagged Ldb16 and plasmid-borne SEl1-FLAG with a photoreactive Bpa at the indicated positions were subjected to UV irradiation. Non-irradiated cells were used as controls. Solubilized membranes were subjected to immunoprecipitation with anti-FLAG antibodies, and bound proteins were analyzed by immunoblotting with FLAG and HA antibodies.

B Schematic representation of the Sei1-Sei1 and Sei1-Ldb16 site-specific photo-crosslinks obtained in (A). C Analysis of LDs in cells with the indicated genotype after staining with the neutral lipid dye BODIPY 493/503. Scale bar corresponds to $5 \mu \mathrm{m}$. D Quantification of LD diameter of cells shown in (C). At least 100 LDs were analysed for a minimum of 3 biological repeats. Red bars represent median diameter. $n=3$. Difference in distribution of LD size was tested using a two sided Kolmogorov-Smirnov test ( ${ }^{\star \star \star \star} p<0.0001$, n.s. non-significant). E Cartoon of Ldb16 In silico structural prediction by trRosetta. Inset shows a short helical element rich in hydroxylated residues (in red). F Analysis of LDs in cells with the indicated genotype after staining with the neutral lipid dye BODIPY493/503. Scale bar corresponds to $5 \mu \mathrm{m}$. G Quantification of LD diameter of cells shown in (F). At least 100 LDs were analysed for a minimum of 3 biological repeats. Red bars represent median diameter. $n=3$. Difference in distribution of LD size was tested using a two sided Kolmogorov-Smirnov test ( ${ }^{\star \star \star \star} p<0.0001$, n.s. non-significant).

by cryo-EM (Fig. $4 \mathrm{~F}$ and Fig. $\mathrm{S} 4 \mathrm{H}-\mathrm{J})$. As predicted by $\mathrm{MD}$, the luminal domains of Sei1 and Sei ${ }^{\Delta \mathrm{LH}}$ were indistinguishable (Fig. 4G). However, in Sei1 ${ }^{\Delta \mathrm{LH}}$ the TM domains could not be resolved (Fig. 4F, G), likely due their increased movement. Together, these data show that the LH has a central role in controlling the positioning and dynamics of Sei1 TM segments, both important for Ldb16 stability. Moreover, our data indicate that Seil luminal and TM domains independently contribute to LD formation.

Seil transmembrane segments contribute to $\mathrm{LD}$ formation. We identified a critical role of Seil TMs in LD formation through the stabilization of Ldb16. Our coarse-grained MD simulations also showed that Sei1 TMs, unlike its luminal domain, are able to interact with TAG (Fig. 2D). Thus, we wondered if Seil TMs, through this potential TAG binding activity, contributed to LD formation independently of Ldb16. To test this possibility, we asked whether Seil could modify LD morphology in the absence of Ldb16. Aberrant LDs observed in sei1 $\Delta, \quad l d b 16 \Delta$ and sei1 1 ldb164 mutant cells are largely indistinguishable ${ }^{18,19,35}$. Similarly, aberrant LD morphology of sei1 $\Delta l d b 16 \Delta$ was unmodified by plasmid-borne Seil expression from its endogenous promoter (Fig. S5A). However, Seil expression from the strong $A D H 1 \mathrm{p}$ resulted in striking changes in LD morphology in sei1 1 ldb16 16 cells (Fig. 5A, B and S5A). While in this condition LDs were still aberrant, they appeared smaller and clustered at the expense of supersized LDs, which were present at much lower frequency (Fig. 5A, B). This result shows that Seil can modify TAG partition into LDs independently of Ldb16. To test whether this activity could be ascribed to Seil TMs, we generated SeilSecTM and Sei1 ${ }^{\text {WALP }}$ mutants, in which Sei1 TM1 and TM2 were replaced respectively, by Sec61 TM1 and a synthetic WALP $\mathrm{TM}^{39}$, composed of alternating leucines and alanines, and flanked by tryptophan residues (Fig. S5B). Strikingly, Seil ${ }^{\mathrm{SecTM}}$ and Sei1 WALP failed to modify LD morphology in seil 1 ldb164 (Fig. 5A, B) as well as in sei1 $\Delta$ cells (Fig. S5C, D). Both Seil ${ }^{\text {SecTM }}$ and Sei1 ${ }^{\text {WALP }}$ were expressed to the expected levels (Fig. S5E) and formed oligomers (Fig. S5F), consistent with the involvement of the luminal domain in Seil ring assembly. This result confirms that Seil luminal domain does not interact with TAG. Moreover, it indicates that Seil TMs contribute to the partition of TAG into LDs.

The swap of TMs in Sei1 ${ }^{\text {SecTM }}$ and Sei1 WALP likely affects the methionine-aromatic interactions with the $\mathrm{LH}$. Thus, besides the amino acid sequence, Sei1 ${ }^{\text {SecTM }}$ and Sei1 ${ }^{\text {WALP }}$ might also differ in the dynamics of the TMs. To identify the critical TM determinants for the TAG interactions we tested Seil ${ }^{\Delta L H}$, a mutant where only TM dynamics is affected. Like WT Seil, expression of Sei1 ${ }^{\Delta \mathrm{LH}}$ triggered dramatic changes in $\mathrm{LD}$ morphology in sei1 $\Delta l d b 16 \Delta$ cells (Fig. 5A, B). Thus, amino acid composition but not dynamics appears to be critical for the interactions of Sei1 TM with TAG. Finally, we tested whether the ability of Seil TMs to interact with TAG is conserved in human Seipin. Remarkably, expression of Sei1 ${ }^{\mathrm{hs} T M}$, a chimeric protein between Seil luminal domain and the TMs of human Seipin (Fig. S5G), triggered LD morphological changes in sei1 $\Delta$ ldb16 4 cells as observed by expression of WT Seil (Fig. 5A, B). As expected, expression of WT human Seipin (WT hs) in seil 1 ldb164 cells restored normal LDs (Fig. 5A, B) ${ }^{18}$. Thus, interactions between TAG and the TMs of Seil, and perhaps other Seipins, contribute to LD formation and morphology.

\section{Discussion}

Seipins have conserved roles in $\mathrm{LD}$ biogenesis but the mechanistic basis for their function is poorly understood. Here, we identify the determinants within the Sei1/Ldb16 Seipin complex essential for proper LD formation. We show that regions both in the membrane and lumen of the ER play critical roles, likely through their ability to interact with TAG (Fig. 6). Besides binding and stabilizing Ldb16, the Sei1 TMs increase the local concentration of TAG in the proximity of the Seipin complex (Fig. 6, Step 1), possibly controlling its access to the homodecameric Seil ring (Fig. 6, Step 2). Once inside the ring, hydroxyl-residues in a short Ldb16 helix interact with TAG (Fig. 6, Step 3), increasing its local concentration and promoting phase separation into a lens like structure. As discussed below, we propose that these sequential TAG interactions are general steps in Seipin-mediated neutral lipid recruitment required for LD formation and lipid homeostasis.

The Seil luminal domain forms a homooligomeric ring, like other Seipins ${ }^{27,28}$. In human Seipin, the ring facilitates TAG concentration via interactions with two highly conserved hydroxyl-containing residues in a central hydrophobic helix ${ }^{28-30}$. In contrast, we showed that the Seil luminal ring lacks a hydrophobic helix and does not concentrate TAG. Instead, we found that Ldb16 complements the Seil luminal domain through a short helical region rich in hydroxyl-residues, thus providing the TAG interacting moieties to the yeast Seipin complex. The ring assembly of Seil luminal domain facilitates this interaction by acting as a scaffold for Ldb16. The ER membrane protein Ldo45 was shown to interact with Sei1/ Ldb16 complex and modulate its activity 33,34 . Moreover, the human Ldo45 homolog, LDAF1/Promethin, interacts with the hydrophobic helix of Seipin luminal domain, an interaction that is modulated by TAG ${ }^{30-32}$. Thus, it is possible that Ldo45 regulates the Sei1/Ldb16 complex by controlling Ldb16 position and/or TAG binding activity.

We identified critical roles for the Seil TMs during LD formation, by binding independently Ldb16 and TAG. The binding to Ldb16 depends on the unique TM1/TM2 arrangement maintained via methionine-aromatic interactions between the $\mathrm{LH}$ and 
A

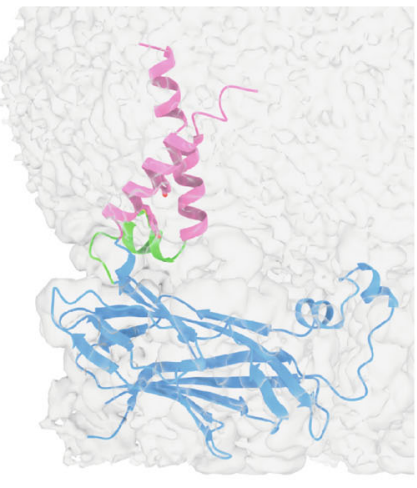

B
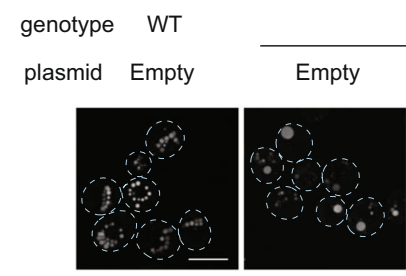

$\operatorname{sei} 1 \Delta$

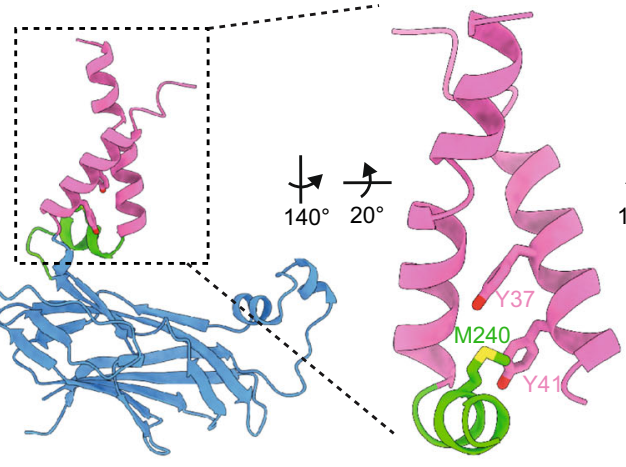

$\mathrm{LH}(\mathrm{a} 3)$

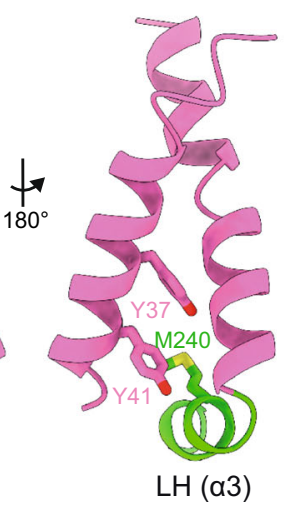

$\mathrm{LH}(\mathrm{\alpha} 3)$
D

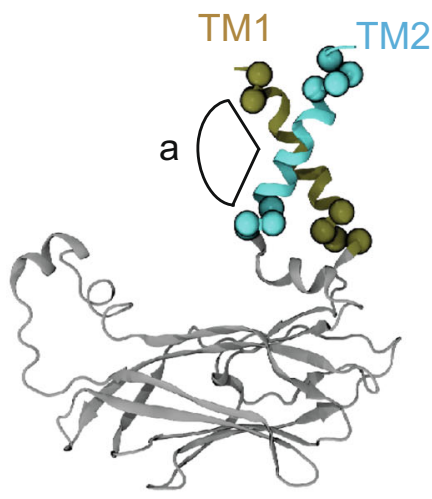

C

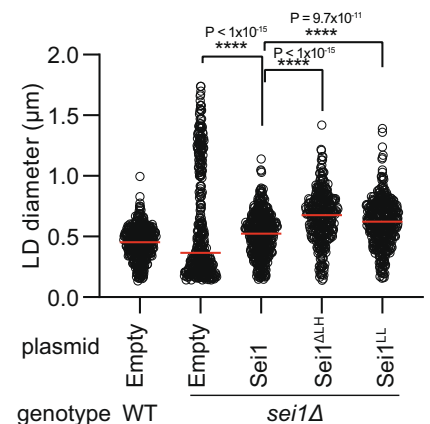

E

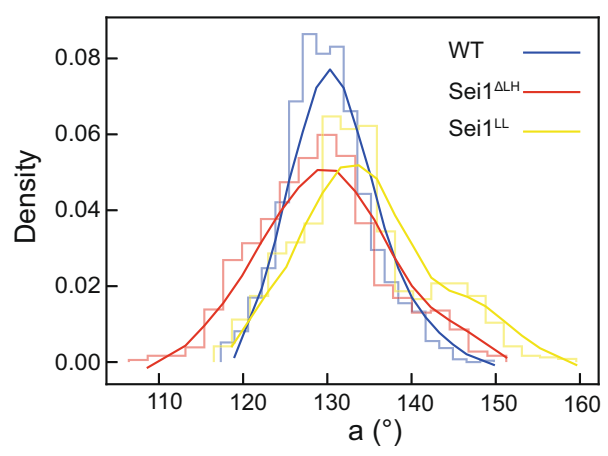

$\mathrm{F}$

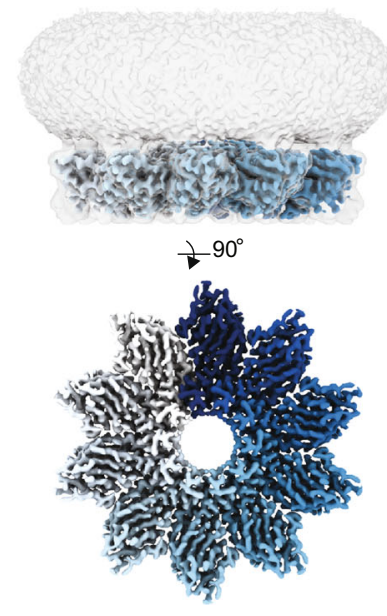

G
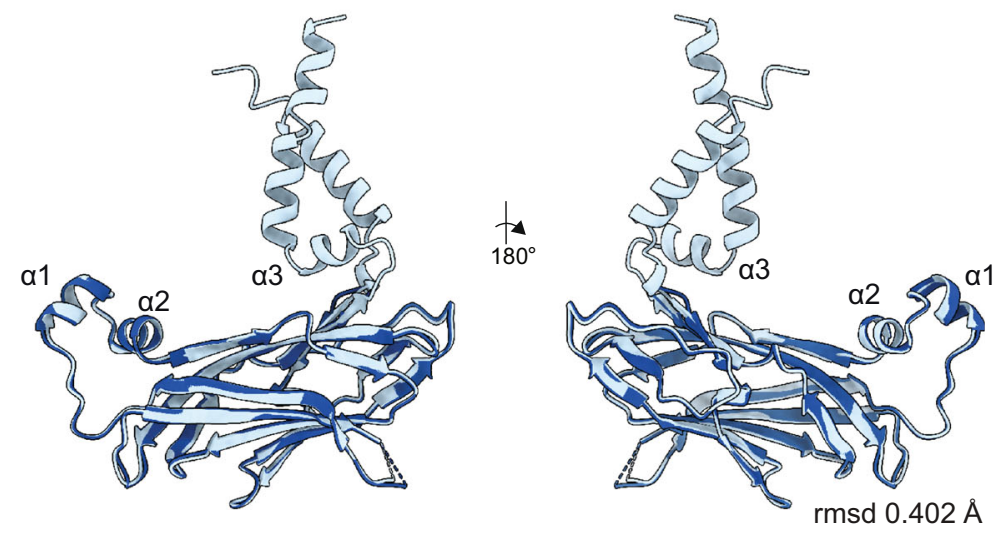

TM1, respectively. While the TMs of human and fly Seipins were not resolved in previous structural studies ${ }^{27,28}$, in silico analysis predicts the existence of a short helical domain, equivalent to the $\mathrm{LH}$, in those Seipins. It will be interesting to test the functional relevance of this helical domain, and whether it regulates the relative arrangement of the TMs in other Seipins.

In addition, Seil TMs also modulate the partitioning of TAG into LDs. By promoting only mild TAG concentration, Sei1 TMs 
Fig. 4 Sei1 Locking Helix positions transmembrane segments and facilitates Ldb16 binding. A Sei1 protomer model shown (left) in the context of the map at low contour (level of 0.005) within detergent micelle or (middle) without map overlay. Inset (right) depicts the locking helix (LH, green) and TM1 and TM2 (pink). The LH M240 (yellow) establishes methionine-aromatic interaction with Y37 and Y41 (red) in TM1. B Analysis of LDs in cells with the indicated genotype after staining with the neutral lipid dye BODIPY 493/503. Scale bar corresponds to $5 \mu$ m. C Quantification of LD diameter of cells shown in (B). At least 100 LDs were analysed for a minimum of 3 biological repeats. Red bars represent median diameter. $n=3$. Difference in distribution of LD size was tested using a two sided Kolmogorov-Smirnov test ${ }^{\star \star \star \star} p<0.0001$, n.s. non-significant). D Schematic representation of the angle (a) between Sei1 TM1 and TM2 analyzed by atomistic MD simulations in (E). E Computed angles between TMs 1 and 2 during $3 \times 120 \mathrm{~ns}$ atomistic MD simulations of Sei1, Sei1 ${ }^{\mathrm{LH}}$ and Sei1 ${ }^{\mathrm{LL}}$ with the first $20 \mathrm{~ns}$ discarded as equilibration. The data has been binned into 1D histograms (thin line) with a line fitted through the bin centers and smoothed using a Savitzky-Golay filter with a step of 5 (thick line). F Cryo-EM map of Sei ${ }^{\Delta \mathrm{LH}}$ homodecamer shown as side view (top), depicting luminal domain protein density (contour level of 0.05) with each Sei1 protomer colored in shades of blue, and the surrounding detergent micelle shown in transparent gray (contour level of 0.005) or 90-degree rotated view looking down from the cytosol (bottom), with detergent micelle omitted for clarity. G Structural alignment of the luminal domains of Sei1 (light blue) and Sei1 $\Delta$ LH (dark blue).

A

\begin{tabular}{|c|c|c|c|c|c|c|c|}
\hline genotype & WT & & & & $1 \Delta / d b 16 \Delta$ & & \\
\hline plasmid & Empty & Empty & Sei1 & Sei1 1 SectM & Sei1 WALP & Sei $1^{\Delta L H}$ & Sei $1^{\text {hsTM }}$ \\
\hline
\end{tabular}

B

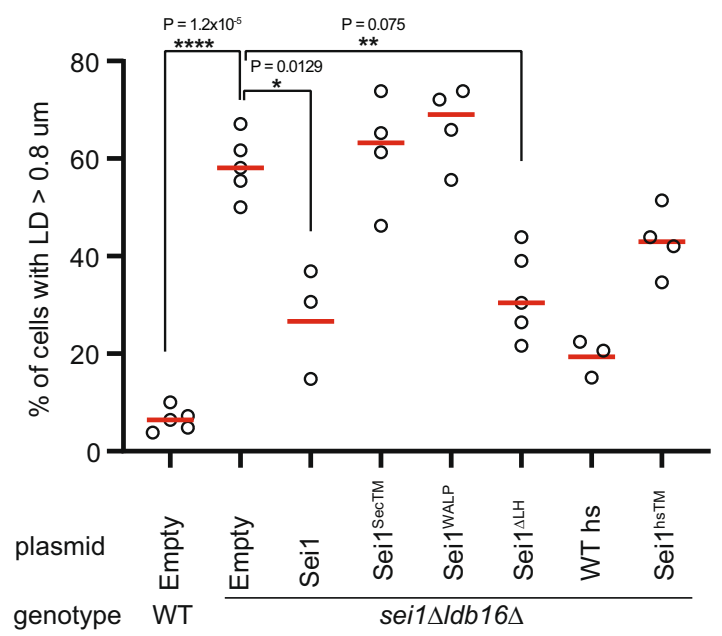

Fig. 5 Sei1 transmembrane segments contribute to LD formation. A Analysis of LDs in cells with the indicated genotype after staining with the neutral lipid dye Bodipy493/503. Scale bar corresponds to $5 \mu \mathrm{m}$. Blue and yellow arrowheads indicate supersized LDs (ssLDs) and LD aggregates, respectively. B Quantification of ssLDs in cells with the indicated genotype. At least 50 cells were analysed per strain for a minimum of 3 biological replicates (WT

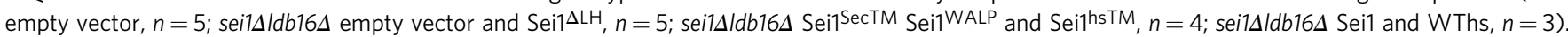
Red bars represent median percentage. Difference in frequency of $\operatorname{ssLDs}$ was tested using a two sided paired t-test $\left({ }^{\star} p<0.05,{ }^{\star \star} p<0.01,{ }^{\star \star \star \star} p<0.0001\right.$, n.s. non-significant).

are incapable to promote lens formation. Instead, they may regulate the kinetics of TAG entry into the Seil ring, as proposed for human Seipin ${ }^{29}$. Such an activity may also be important to coordinate lens growth with incorporation of monolayer phospholipids during LD expansion. Seipin rings not in contact with lenses are highly mobile in the $\mathrm{ER}^{17,20,40}$. Thus, by encountering TMs of dynamic Seipin rings, TAG molecules dispersed in the membrane may be concentrated at specific ER regions for proper LD assembly, a function consistent with the role of Seipin in averting lipotoxicity. Besides TAG, Sei1 TMs may also affect the distribution of other lipids in the ER bilayer. Consistent with this possibility, in silico predictions indicate that the TMs of human Seipin promote localized enrichment of diacylglycerol (DAG) and phosphatidylethanolamine $(\mathrm{PE})^{29}$. Given the impact of ER bilayer composition in LD biogenesis ${ }^{16,41-44}$, Seipins TMs can contribute for a local membrane environment conductive to proper LD formation. Our findings provide a mechanistic framework for Seipin function, which will guide future structural and biophysical studies.

\section{Methods}

Reagents. BODIPY493/503 was purchased from Invitrogen. Antibodies used in this study were anti -FLAG M2-Peroxidase (HRP), Clone M2-A8592 (Sigma Aldrich) product number A8592, anti-HA High affinity (clone 3F10) product number 11867431001 (Roche), PGK1 Monoclonal (22C5D8) product number 459250 (Invitrogen), DPM1 monoclonal (5C5A7) product number A6429 


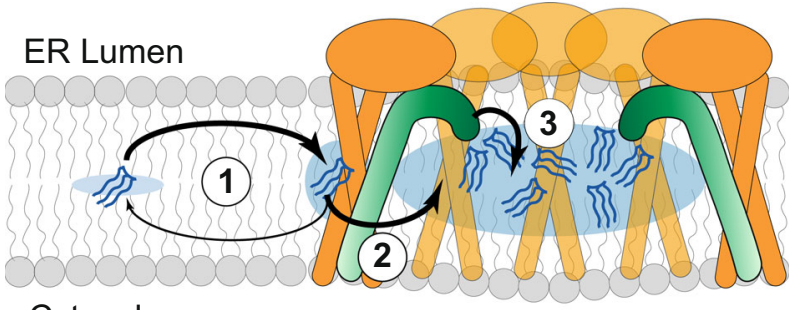

Cytosol

\section{Sei1 $\square$ Ldb16 coalesced TAG קر TAG molecule}

Fig. 6 A model for LD formation by the Sei1-Ldb16 complex. Sequential TAG interactions mediate LD assembly by the Sei1-Ldb16 complex. In the ER bilayer, TAG molecules (blue) concentrate in proximity of Seipin oligomers (orange) via weak interaction with Sei1 TMs. TAG molecules within the ring interact strongly with Ldb16 (green) hydroxyl-containing residues, facilitating TAG coalescence and lens formation (see text for details).

(Thermo Fisher Scientific)). Polyclonal anti-Seil (rabbit), anti-Ldb16 (rabbit) antibodies were previously described ${ }^{33}$.

Yeast strains and plasmids. The strains used are isogenic either to BY4741

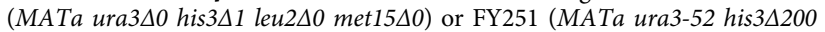
leu2 $\Delta 1 \operatorname{trp1\Delta 63)}$ and are listed in the Table S1. Tagging of proteins and individual gene deletions were performed by standard PCR-based homologous recombination ${ }^{45}$ and standard yeast molecular genetics protocols ${ }^{46}$.

Plasmids used are based on pRS316, pRS416 pRS423 or pRS426 $6^{47,48}$ and listed in Table S2. 3xFLAG-tagged Seil was expressed from the native Seil promotor (494 bp upstream of the SEII ORF) or an $A D H 1$ promotor, and followed by the $A D H 1$ terminator. 3xFLAG-tagged Ldb16 was expressed from the native promotor ( 459 bp upstream of $L D B 16$ ORF) followed by the $A D H 1$-terminator. Primers used in this study are listed in Table S3.

Culture conditions. Cells were cultured in synthetic defined glucose media (SD), unless otherwise indicated. SD contained per liter: $6.7 \mathrm{~g}$ yeast nitrogen base with ammonium sulfate (YNB; MP biomedicals), $0.6 \mathrm{~g}$ complete supplement mixture without histidine, leucine, tryptophan, and uracil (CSM-HIS-LEU-TRP-URA; MP biomedicals), and $20 \mathrm{~g}$ glucose (Sigma-Aldrich). Media was supplemented with histidine $(60 \mu \mathrm{M})$, leucine $(1.68 \mathrm{mM})$, uracil $(0.2 \mathrm{mM})$ and tryptophan $(0.4 \mathrm{mM})$ as required. For inositol free media, YNB devoid of inositol (MP biomedical) was used.

All cultures were incubated at $30^{\circ} \mathrm{C}$ with shaking at $200 \mathrm{rpm}$. Culture density was determined by measuring turbidity at $600 \mathrm{~nm}\left(\mathrm{OD}_{600}\right)$ using a GENESYS $10 \mathrm{~S}$ UV-VIS spectrophotometer (Thermo Scientific).

Protein expression and purification for structural analysis. For protein overexpression, sei1 $\Delta l d b 16 \Delta$ mutants were transformed with a plasmid encoding Sei1FLAG, Sei1 ${ }^{\Delta L H}$-FLAG or both Sei1-FLAG and Ldb16-SBP ${ }^{49}$. Cells were grown and protein was expressed as described ${ }^{50}$. Cell pellets $(\sim 150 \mathrm{~g})$ were harvested by centrifugation, washed with water and lysis buffer (50 mM Tris.HCl [pH7.4], $200 \mathrm{mM} \mathrm{NaCl}, 1 \mathrm{mM}$ EDTA). Cells were resuspended in $100 \mathrm{~mL}$ of lysis buffer with $1 \mathrm{mM}$ phenylmethylsulfonylfluoride (PMSF) (Roche) and $1.5 \mu \mathrm{M}$ of Pepstatin A (Sigma-Aldrich) and transferred to a bead beater chamber (BioSpec) containing $\sim 150$ g glass beads $(0.5 \mathrm{~mm}$ diameter; BioSpec). Bead beater chamber was assembled with an ice water jacket. Lysis was induced by 40 cycles of $30 \mathrm{~s}$ on/off. Glass beads were removed by filtration and lysates cleared by low-speed spinning at 2000 $\mathrm{g}$ for $30 \mathrm{~min}$. Total membrane fraction was prepared by centrifugation $(185511.4 \times \mathrm{g}$ in a Ti-45 for $45 \mathrm{~min})$. and washed with lysis buffer. The membrane pellet was solubilized for $4 \mathrm{~h}$ in $195 \mathrm{~mL}$ of lysis buffer supplemented with $1 \%(\mathrm{w} / \mathrm{v})$ of DDM (Anatrace) and 0.1\% (w/v) CHS (Anatrace), $1 \mathrm{mM}$ PMSF (Roche), $1.5 \mu \mathrm{M}$ Pepstatin A (Sigma-Aldrich). Non-solubilized material was removed by centrifugation ( $185511.4 \times \mathrm{g}$ in a Ti- 45 for $30 \mathrm{~min}) .4 \mathrm{~mL}$ of FLAG matrix $-\mathrm{M} 2$ affinity gel - A2220 (Sigma Aldrich) was added to the solubilized membranes and incubated at $4{ }^{\circ} \mathrm{C}$ overnight. After incubation, the material was transferred to $20 \mathrm{~mL}$ gravity columns and beads were washed with 10 column volumes of Akta buffer (50 mM Tris.HCl [pH7.4], $200 \mathrm{mM} \mathrm{NaCl}, 1 \mathrm{mM}$ EDTA, 0.015\% DDM, 0.0015\% CHS) by gravity flow. Bound proteins were eluted with Akta buffer in $5 \times 3 \mathrm{~mL}$ fractions containing $0.2 \mu \mathrm{g} / \mathrm{mL} 3 \times \mathrm{FLAG}$-peptide for the first two fractions and $0.4 \mu \mathrm{g} / \mathrm{mL} 3 \times \mathrm{xLAG}$-peptide for the last three rounds. Eluted material was concentrated using $100 \mathrm{kDa}$ cut off centrifugal filters (Amicon Ultra, Merck) until the volume reached below $2 \mathrm{~mL}$. The concentrated material was run with an AKTA
Pure (SEC) (GE Healthcare) over a $24 \mathrm{~mL}$ Superose 6 10/300 GL size exclusion column in Akta buffer, at $0.5 \mathrm{~mL} / \mathrm{min}$, collecting $1 \mathrm{~mL}$ aliquots

Negative stain. For negative stain EM analysis, $8 \mu \mathrm{L}$ of purified Seil (protein concentration $\sim 20 \mathrm{ng} / \mu \mathrm{L}$ ) was added to glow discharged 300 mesh carbon support films (TAAB) and immersed in $20 \mu \mathrm{L}$ water, twice in $20 \mu \mathrm{L}$ of $2 \%$ uranyl acetate, and dried for at least $5 \mathrm{~min}$ before use. Grids were imaged in a FEI Tecnai T12 transmission electron microscope.

Cryo-EM sample preparation and data acquisition. Four microliters of purified Seil or Seil-Ldb16 complex at a concentration of $4 \mathrm{mg} / \mathrm{mL}$ was adsorbed to glowdischarged gold UltrAufoil grids ( 300 mesh, R1.2/1.3) for $10 \mathrm{~s}$. Grids were then blotted for $2 \mathrm{~s}$ at $100 \%$ humidity at $9^{\circ} \mathrm{C}$ and frozen in liquid ethane using a Vitrobot Mark IV (Thermo Fisher Scientific). Data were collected in counting mode on a Titan Krios G3 (FEI) operating at $300 \mathrm{kV}$ with a GIF energy filter (Gatan) and K2 Summit detector (Gatan) using a pixel size of $0.822 \AA$, a dose rate of $6 \mathrm{e}^{-}$per $\AA^{2}$ per s and an exposure of $8 \mathrm{~s}$, corresponding to a total dose of $48 \mathrm{e}^{-}$ per $\AA^{2}$ collected over 32 fractions.

Four microliters of purified Sei1 $\Delta 231-243\left(\mathrm{Sei}^{\Delta \mathrm{LH}}\right)$ at a concentration of $7.8 \mathrm{mg} / \mathrm{ml}$ was adsorbed to a glow-discharged gold UltrAufoil grid (300 mesh, R1.2/1.3) for $10 \mathrm{~s}$. Grids were then blotted for $2 \mathrm{~s}$ at $100 \%$ humidity at $6{ }^{\circ} \mathrm{C}$ and frozen in liquid ethane using a Vitrobot Mark IV (Thermo Fisher Scientific). Data were collected in counted super-resolution mode on a Titan Krios G3 (FEI) operating at $300 \mathrm{kV}$ with a BioQuantum imaging filter (Gatan) and $\mathrm{K} 3$ direct detection camera (Gatan) at $105,000 \times$ magnification, physical pixel size of $0.832 \AA$. Data were collected at a dose rate of $22.2 \mathrm{e}-$ per $\AA^{2}$ per $\mathrm{s}$ and an exposure time of $2.66 \mathrm{~s}$, corresponding to a total dose of $59.1 \mathrm{e}-$ per $\AA 2$ collected over 40 fractions.

Cryo-EM data processing. Initial micrograph processing was performed in real time using the SIMPLE pipeline ${ }^{51}$, using SIMPLE-unblur for patched motion correction, SIMPLE-CTFFIND for CTF estimation and SIMPLE-picker for particle picking. Resolution estimates were derived from gold-standard Fourier shell correlations (FSCs) using the 0.143 criterion as calculated within RELION-3.1 ${ }^{52}$. Local resolution estimations were calculated within RELION-3.1.

1,369,344 combined particles from Seil (2,008 movies) and Seil-Ldb16 (6,499 movies) datasets were extracted in $300 \times 300$ pixel boxes and subjected to initial $2 \mathrm{D}$ classification (SIMPLE_cleanup2D) to remove junk particles. A subset of 81,812 recovered particles were used to generate a C10-symmetrized ab initio model (SIMPLE_initial_3Dmodel). All further downstream processing was performed in RELION-3.1 $1^{52}$. The initial model was lowpass filtered to $60 \AA$ and used as reference for unmasked 3D classification ( $7.5^{\circ}$ sampling, 15 iterations, 3 classes,

C10 symmetry) against the same particle subset. The map corresponding to the class with highest particle distribution (73.9\% of total particles) was lowpass filtered to $40 \AA$ and used as reference for unmasked 3D classification ( $7.5^{\circ}$ sampling, 15 iterations, 3 classes, C10 symmetry) using the cleaned particle subset. Particles $(234,989)$ and map belonging to the dominant class $(46.1 \%$ particle distribution) were subjected to 3D auto-refinement in C10 using a $15 \AA$ lowpass filter for the reference map and a mask encompassing all protein and detergent density. This generated a volume with global resolution estimate of $3.3 \AA$ A Per-particle defocus refinement and beamtilt estimation followed by Bayesian particle polishing in a larger box $(432 \times 432)$ further improved map quality to $2.7 \AA$. Seil and Seil-Ldb16 datasets were initially processed independently until it became apparent that volumes generated from refinements for either datasets were identical, with no additional density corresponding to Ldb16. To boost the number of particles going into the final reconstructions and improve map quality, the two datasets were combined and processed together. Data processing workflow is presented in Fig. S1D

In total $1,198,818$ particles from the Seil ${ }^{\Delta \mathrm{LH}}$ dataset $(7,077$ movies) were extracted in $300 \times 300$ pixel boxes and subjected to initial $2 \mathrm{D}$ classification (SIMPLE_cleanup2D) to remove junk particles followed by further processing in RELION-3.152. Unmasked 3D classification ( $7.5^{\circ}$ sampling, 15 iterations, 4 classes, C1 symmetry) was performed using the cleanup2D-recovered particles $(431,932)$, against a $40 \AA$ lowpass filtered Seil reference map. 3D auto-refinement (in C1) with a mask encompassing all protein and detergent was performed independently on the two most populated classes, resulting in $4.0 \AA$ and $4.5 \AA$ maps, respectively. No significant differences in map density were observed across both refined volumes. Particles belonging to both classes were therefore combined (260,532 total particles), reextracted and recentred in $432 \times 432$ boxes, and subjected to masked 3D auto-refinement with C10 symmetry, resulting in a $3.3 \AA$ map. Data processing workflow is presented in Fig. S4H.

Model building and refinement. The atomic model of Seil (residues 24-132, 145-265; Table S4) was built de novo from the $2.7 \AA$ local-resolution filtered and sharpened map following several rounds of manual building using Coot v. $0.944^{53}$ and real-space refinement in PHENIX v.1.18.2-387445 ${ }^{54}$ using secondary structure, NCS, rotamer, and Ramachandran restraints.

The atomic model of Sei1 ${ }^{\Delta \mathrm{LH}}$ (residues 49-132, 145-230; Table S4) was generated by rigid-body fitting the Seil model into the $3.3 \AA$ Sei1 ${ }^{\Delta \mathrm{LH}}$ localresolution filtered and sharpened map followed by manual building in Coot and 
multiple rounds of real-space refinement in both Coot and PHENIX. Both Seil and Sei1 ${ }^{\Delta \mathrm{LH}}$ models were validated using MolProbity ${ }^{55}$ within PHENIX. Figures were prepared using UCSF ChimeraX v.1.149, and PyMOL v.2.4.0. Structural alignments of yeast Seil and Seil ${ }^{\Delta \mathrm{LH}}$ protomer models were performed within ChimeraX using the MatchMaker command. Structural alignments of yeast Seil protomers (residues 49-232) with protomers of fly (PDB 6MLU; residues 88-240) or human (PDB 6DS5; residues 60-219) Seipin were performed within CCP4 ${ }^{56}$ using SSM superposition within the superpose ${ }^{57}$ program.

Molecular dynamics simulations. For the coarse-grained MD, the coordinates of yeast Seil were converted to the Martini 2.2 force field ${ }^{58,59}$. Alternatively, the human Seipin luminal domain (PDB 6DS5) was used, with the TM regions modeled as per the yeast Seil TMs, using Swiss-Model ${ }^{60}$, with the final model available at osf.io/5depa. Harmonic bonds of $500 \mathrm{~kJ} \mathrm{~mol}^{-1} \mathrm{~nm}^{-2}$ were applied between all protein backbone beads within $1 \mathrm{~nm}$. For the human Seipin simulations, additional flat-bottomed distance restraints of $1000 \mathrm{~kJ} \mathrm{~mol}^{-1} \mathrm{~nm}^{-2}$ were applied using PLUMED 2.4.4 $4^{61}$ between the backbone beads of Val-248 of one protomer, with Leu-39, Leu-167, and Val-248 of the next protomer.

Proteins were embedded into membranes composed of 3\% TAG (trioleylglycerol) and 97\% POPC, using the insane protocol ${ }^{62}$. All systems were solvated with Martini waters and $\mathrm{Na}^{+}$and $\mathrm{Cl}^{-}$ions to a neutral charge and a $0.15 \mathrm{M}$ concentration. Systems were minimized using the steepest descents method, followed by $1 \mathrm{~ns}$ equilibration with $5 \mathrm{fs}$ time steps, then by $100 \mathrm{~ns}$ equilibration with $20 \mathrm{fs}$ time steps, before $5 \mathrm{x}$ ca. $13 \mu$ s (yeast) or $3 \times 5 \mu$ s (human) production simulations using $20 \mathrm{fs}$ time steps, all in the NPT ensemble with the V-rescale thermostat and semi-isotropic Parrinello-Rahman pressure coupling ${ }^{63,64}$.

For the atomistic simulations, short CG sims were run of the yeast Seil complex in a POPC membrane, either using the WT system or with the mutations added manually. Snapshots were then converted to an atomistic description using the cg2at program ${ }^{65}$, before production simulations of $3 \times 120 \mathrm{~ns}$ per system using a 2 fs time step in the NPT ensemble with the V-rescale thermostat and semiisotropic Parrinello-Rahman pressure coupling ${ }^{63,64}$.

All simulations were run in Gromacs ${ }^{66} .2 \mathrm{D}$ densities were computed using gmx density from the Gromacs software, and plotted in Matplotlib ${ }^{67}$. Images were made in $\mathrm{VMD}^{68}$.

In vivo site-specific crosslinking. Site specific crosslinking was conducted as previously described ${ }^{69}$. Briefly, seil $\Delta$ cells were transformed with two plasmids, one encoding both for a modified tRNA synthetase capable of charging the unnatural amino acid benzoyl phenylalanine (BPA) on a tRNA as well as amber stop codon suppressor tRNA, and a second plasmid encoding ADH1-promotor expressed Seil-FLAG with individual amber codons. Cells carrying both plasmids were precultured in $\mathrm{SD}$ for $8 \mathrm{~h}$, transferred to $100 \mathrm{~mL}$ of the same media supplemented with $\mathrm{BPA}$ to a final concentration of $0.3 \mathrm{mM}$ (from a $0.3 \mathrm{M}$ in $1 \mathrm{M} \mathrm{NaOH}$ freshly prepared stock) and cultured to mid-exponential phase (OD 1). Cells were harvested by a centrifugation for $2 \mathrm{~min}$ at $3000 \mathrm{~g}$ and resuspended in $2 \mathrm{~mL}$ of cold water. Half of cells were transferred to a 12 well plate and subjected to UV irradiation for $1 \mathrm{~h}$ at $4{ }^{\circ} \mathrm{C}$ using a B-100AP lamp (UVP, CA). The other half of the cells was incubated on ice and served as non-irradiated control. After UV irradiation, cells were harvested by centrifuge spin for $2 \mathrm{~min}$ at $3000 \mathrm{~g}$. Both irradiated and control cells were lysed in Lysis buffer (50 mM Tris.HCl [pH7.4], $200 \mathrm{mM} \mathrm{NaCl}$, $1 \mathrm{mM}$ EDTA, $1 \mathrm{mM}$ PMSF (Roche) and 1x cOmplete protease inhibitor cocktail (Roche)by 5-6 $\times 1 \mathrm{~min}$ cycles of bead beating. Lysates were cleared by a $10 \mathrm{~min}$ centrifugation at $600 \mathrm{~g}$. Cleared lysates were centrifuged at $100,000 \mathrm{~g}(25 \mathrm{~min}$ at $4{ }^{\circ} \mathrm{C}$ ) in an Optima Max Tabletop Ultracentrifuge in a TLA 45 rotor (Beckman Coulter) to obtain crude membrane fractions. The membrane pellet was resuspended in denaturing buffer (50 mM Tris.HCl [pH7.4], 1 mM EDTA, $1 \%$ SDS, $2 \mathrm{M}$ urea) and solubilized at $65^{\circ} \mathrm{C}$ for $30-40 \mathrm{~min}$ with vigorous shaking. Insolubilized material was pelleted by centrifugation $(15 \mathrm{~min}, 13000 \mathrm{~g})$. The solubilized material was diluted with lysis buffer supplemented with $1 \%$ Nonidet P-40 and incubated overnight with anti-FLAG M2 magnetic beads-m8823 (Sigma-Aldrich). Beads were washed 3 times with lysis buffer/1\% Nonidet P- 40 and bound proteins eluted with SDS buffer and analyzed by immunoblotting.

Native Immunoprecipitation. Cells were in SD until med-exponential phase (OD $\sim 1$ ). Cells corresponding to $50 \mathrm{OD}$ were then harvested by centrifugation at $3000 \mathrm{~g}$ and washed with lysis buffer (50 mM Tris. $\mathrm{HCl}$ [pH7.4], $200 \mathrm{mM} \mathrm{NaCl}, 1 \mathrm{mM}$ EDTA, $2 \mathrm{mM}$ phenylmethylsulfonyl fluoride and $1 \times$ cOmplete protease inhibitor cocktail). Lysates and crude membrane fractions were prepared as described above. Detergent extracts were prepared by solubilizing crude membrane fractions in lysis buffer/1\% decyl maltose neopentyl glycol (DMNG). Insolubilized material was cleared by centrifugation $(20,000 \mathrm{~g}, 15 \mathrm{~min})$. The cleared detergent extracts were incubated overnight at $4{ }^{\circ} \mathrm{C}$ with FLAG M2 magnetic beads-m8823 (SigmaAldrich). Beads were washed 3 times with lysis buffer/1\% DMNG, eluted with SDSPAGE sample buffer and analyzed by immunoblotting. The input corresponds to $10 \%$ of the total extract used for IP.

Gel electrophoresis and immunoblotting. For protein quantification of whole cell lysates, cells were lysed using $\mathrm{NaOH}$ as described previously ${ }^{70}$. Briefly, cells pellets corresponding to $1 \mathrm{OD}$ were suspended in $0.15 \mathrm{M} \mathrm{NaOH}$ and incubated on ice for $10 \mathrm{~min}$. Cells were pelleted, and resuspended in Laemmli sample buffer ${ }^{71}$ and incubated $65^{\circ} \mathrm{C}$ for $10 \mathrm{~min}$ with vigorous shaking. Debris was pelleted by a short spin, and samples were loaded on a $4-20 \%$ gradient SDS-polyacrylamide gel, separated by electrophoresis and blotted to a PVDF membrane.

Fluorescence microscopy analysis of LDs. For microscopy analysis of LDs, cells were in synthetic glucose media without inositol to stationary phase. Strains were inoculated at OD 0.1 and cultured for 20-24 h. Lipid droplets were visualized with BODIPY $493 / 503(1 \mu \mathrm{g} / \mathrm{mL})$.

Super resolution fluorescence microscopy was performed on an Olympus IX-83 inverted frame confocal microscope, equipped with a Yokogawa CSU-W1 SoRa super-resolution spinning disc module, and a Photometrics Prime BSI camera. Images were acquired using an UplanApo $60 \times$ objective (N.A. 1.50). Total magnification was $192 \times$. BODIPY was excited using a $488 \mathrm{~nm}$ solid state laser (OBIS) at 5\% intensity, and fluorescence emission was selected using a 525/50 nm bandpass filter. Images were processed for super resolution and deconvoluted (constrained iterative, maximum likelihood, 5 iterations) using Olympus cellSens Dimension software (version 3.1.1, build 21264). Figure preparation and quantification of LD size was done using ImageJ (version 1.53c; National Institutes of Health, USA).

Lipid droplet biogenesis assay. For induction of LD formation, strains were cultures in synthetic defined raffinose media (as SD but with $2 \%$ raffinose as carbon source). Overnight pre-cultures were inoculated at OD 0.25 and cultured for $3 \mathrm{~h}$. Galactose was added to a final concentration of $2 \%$ to induce DGA1 expression. LD formation was followed by staining with BODIPY and imaging by fluorescence microscopy.

Fluorescence microscopy was performed using a Zeiss Axio Observer.Z1 equipped with a Hamamatsu Orca Flash 4.0 digital CMOS camera. Images were acquired using an A Plan-APOCHROMAT 100x objective (N.A. 1.4). BODIPY fluorescence was analysed using a GFP-fluorescence set up consisting of a 485/20 bandpass excitation-filter (Zeiss), a 410/504/582/669-Di01 quad dichroic mirror, and a 525/30 bandpass emission filter. Microscope was controlled using Slidebook 6.0 software (3i).

Statistics and reproducibility. Statistical significance and $p$-values were calculated in GraphPad Prism 7 using the Kolmogorov-Smirnov test (2-tailed) or paired t-testing (2-tailed). Graphs were plotted in Prism. The following figure panels show representative data from at least three independent biological replicates that showed similar results: Figs. 3A, C, F, 4B, 5A, Extended Data Figs. S3D, G, H, S4A, $\mathrm{B}, \mathrm{C}, \mathrm{S} 5 \mathrm{~A}, \mathrm{C}, \mathrm{E}$. The following figure panels show representative data from at least two independent biological replicates that showed similar results: Extended Data Fig. S3F and I.

Reporting summary. Further information on research design is available in the Nature Research Reporting Summary linked to this article.

\section{Data availability}

Coordinates for the structures have been deposited in the Protein Data Bank under accession codes PDB 7OXP (Sei1) and 7OXR (Sei1 $\triangle \mathrm{LH}$ ). The electron microscopy volumes have been deposited in the Electron Microscopy Data Bank under accession codes EMD-13103 (Seil) and EMD-13104 (Seil $\triangle \mathrm{LH})$. The following protein structures were obtained from the Protein Data Bank - PDB 6MLU (fly seipin) and PDB 6DS5 (human Seipin)

\section{Code availability}

All codes used in this study are listed below and freely available as detailed.

Gromacs 2019.4 - https://www.gromacs.org/

VMD 1.9.4 - https://www.ks.uiuc.edu/Research/vmd/

Matplotlib 3.4.2 - https://numpy.org/

NumPy 1.16 .2 - https://www.mdanalysis.org/

Received: 18 July 2021; Accepted: 21 September 2021;

Published online: 08 October 2021

\section{References}

1. Walther, T. C. \& Farese, R. V. Lipid droplets and cellular lipid metabolism. Annu. Rev. Biochem. 81, 687-714 (2012).

2. Olzmann, J. A. \& Carvalho, P. Dynamics and functions of lipid droplets. Nat Rev. Mol. Cell Biol. 20, 137-155 (2019).

3. Krahmer, N., Farese, R. V. \& Walther, T. C. Balancing the fat: lipid droplets and human disease. EMBO Mol. Med. 5, 973-983 (2013). 
4. Tauchi-Sato, K., Ozeki, S., Houjou, T., Taguchi, R. \& Fujimoto, T. The surface of lipid droplets is a phospholipid monolayer with a unique fatty acid composition. J. Biol. Chem. 277, 44507-44512 (2002).

5. Fujimoto, T. \& Parton, R. G. Not just fat: the structure and function of the lipid droplet. Cold Spring Harb. Perspect. Biol. 3, (2011).

6. Bersuker, K. et al. A proximity labeling strategy provides insights into the composition and dynamics of lipid droplet proteomes. Dev. Cell 44, 97-112 (2018). e7.

7. Currie, E. et al. High confidence proteomic analysis of yeast LDs identifies additional droplet proteins and reveals connections to dolichol synthesis and sterol acetylation. J. Lipid Res. 55, 1465-1477 (2014).

8. Walther, T. C., Chung, J. \& Farese, R. V. Lipid droplet biogenesis. Annu. Rev. Cell Dev. Biol. 33, 491-510 (2017).

9. Renne, M. F., Klug, Y. A. \& Carvalho, P. Lipid droplet biogenesis: a mystery "unmixing"? Semin. Cell Dev. Biol. 108, 14-23 (2020).

10. Jacquier, N. et al. Lipid droplets are functionally connected to the endoplasmic reticulum in Saccharomyces cerevisiae. J. Cell Sci. 124, 2424-2437 (2011).

11. Kassan, A. et al. Acyl-CoA synthetase 3 promotes lipid droplet biogenesis in ER microdomains. J. Cell Biol. 203, 985-1001 (2013).

12. Sandager, L. et al. Storage lipid synthesis is non-essential in yeast. J. Biol. Chem. 277, 6478-6482 (2002).

13. Thiam, A. R. \& Forêt, L. The physics of lipid droplet nucleation, growth and budding. Biochim. Biophys. Acta 1861, 715-722 (2016).

14. Choudhary, V., Ojha, N., Golden, A. \& Prinz, W. A. A conserved family of proteins facilitates nascent lipid droplet budding from the ER. J. Cell Biol. 211, 261-271 (2015).

15. Thiam, A. R. \& Ikonen, E. Lipid droplet nucleation. Trends Cell Biol. 31, 108-118 (2021).

16. Chorlay, A. et al. Membrane asymmetry imposes directionality on lipid droplet emergence from the ER. Dev. Cell 50, 25-42 (2019). e7.

17. Wang, H. et al. Seipin is required for converting nascent to mature lipid droplets. Elife 5, (2016).

18. Wang, C.-W., Miao, Y.-H. \& Chang, Y.-S. Control of lipid droplet size in budding yeast requires the collaboration between Fld1 and Ldb16. J. Cell Sci. 127, 1214-1228 (2014).

19. Grippa, A. et al. The seipin complex Fld1/Ldb16 stabilizes ER-lipid droplet contact sites. J. Cell Biol. 211, 829-844 (2015).

20. Salo, V. T. et al. Seipin regulates ER-lipid droplet contacts and cargo delivery. EMBO J. 35, 2699-2716 (2016).

21. Fei, W. et al. Fld1p, a functional homologue of human seipin, regulates the size of lipid droplets in yeast. J. Cell Biol. 180, 473-482 (2008).

22. Magré, J. et al. Identification of the gene altered in Berardinelli-Seip congenital lipodystrophy on chromosome 11q13. Nat. Genet. 28, 365-370 (2001).

23. Szymanski, K. M. et al. The lipodystrophy protein seipin is found at endoplasmic reticulum lipid droplet junctions and is important for droplet morphology. Proc. Natl Acad. Sci. USA 104, 20890-20895 (2007).

24. Boutet, E. et al. Seipin deficiency alters fatty acid Delta9 desaturation and lipid droplet formation in Berardinelli-Seip congenital lipodystrophy. Biochimie 91, 796-803 (2009).

25. Salo, V. T. et al. Seipin facilitates triglyceride flow to lipid droplet and counteracts droplet ripening via endoplasmic reticulum contact. Dev. Cell 50, 478-493 (2019). e9.

26. Lundin, C. et al. Membrane topology of the human seipin protein. FEBS Lett. 580, 2281-2284 (2006).

27. Yan, R. et al. Human SEIPIN binds anionic phospholipids. Dev. Cell 47, 248-256 (2018). e4

28. Sui, X. et al. Cryo-electron microscopy structure of the lipid droplet-formation protein seipin. J. Cell Biol. 217, 4080-4091 (2018).

29. Zoni, V. et al. Seipin accumulates and traps diacylglycerols and triglycerides in its ring-like structure. Proc. Natl Acad. Sci. USA 118, (2021).

30. Prasanna, X. et al. Seipin traps triacylglycerols to facilitate their nanoscale clustering in the endoplasmic reticulum membrane. PLoS Biol. 19, e3000998 (2021).

31. Chung, J. et al. LDAF1 and seipin form a lipid droplet assembly complex. Dev. Cell 51, 551-563 (2019). e7.

32. Castro, I. G. et al. Promethin is a conserved seipin partner protein. Cells 8, (2019).

33. Teixeira, V. et al. Regulation of lipid droplets by metabolically controlled Ldo isoforms. J. Cell Biol. 217, 127-138 (2018).

34. Eisenberg-Bord, M. et al. Identification of seipin-linked factors that act as determinants of a lipid droplet subpopulation. J. Cell Biol. 217, 269-282 (2018).

35. Han, S., Binns, D. D., Chang, Y.-F. \& Goodman, J. M. Dissecting seipin function: the localized accumulation of phosphatidic acid at ER/LD junctions in the absence of seipin is suppressed by $\operatorname{Seil} \mathrm{p}(\Delta \mathrm{Nterm})$ only in combination with Ldb16p. BMC Cell Biol. 16, 29 (2015).

36. Xu, S., Benoff, B., Liou, H.-L., Lobel, P. \& Stock, A. M. Structural basis of sterol binding by NPC2, a lysosomal protein deficient in Niemann-Pick type C2 disease. J. Biol. Chem. 282, 23525-23531 (2007).
37. Chin, J. W. et al. An expanded eukaryotic genetic code. Science 301, 964-967 (2003).

38. Yang, J. et al. Improved protein structure prediction using predicted interresidue orientations. Proc. Natl Acad. Sci. USA 117, 1496-1503 (2020).

39. Killian, J. A. et al. Induction of nonbilayer structures in diacylphosphatidylcholine model membranes by transmembrane alpha-helical peptides: importance of hydrophobic mismatch and proposed role of tryptophans. Biochemistry 35, 1037-1045 (1996).

40. Choudhary, V., El Atab, O., Mizzon, G., Prinz, W. A. \& Schneiter, R. Seipin and Nem1 establish discrete ER subdomains to initiate yeast lipid droplet biogenesis. J. Cell Biol. 219, (2020).

41. Chorlay, A. \& Thiam, A. R. An asymmetry in monolayer tension regulates lipid droplet budding direction. Biophys. J. 114, 631-640 (2018).

42. Choudhary, V. et al. Architecture of lipid droplets in endoplasmic reticulum is determined by phospholipid intrinsic curvature. Curr. Biol. 28, 915-926 (2018). e9.

43. Ben M'barek, K. et al. ER membrane phospholipids and surface tension control cellular lipid droplet formation. Dev. Cell 41, 591-604 (2017). e7.

44. Zoni, V. et al. Pre-existing bilayer stresses modulate triglyceride accumulation in the ER versus lipid droplets. Elife 10, (2021).

45. Longtine, M. S. et al. Additional modules for versatile and economical PCRbased gene deletion and modification in Saccharomyces cerevisiae. Yeast 14, 953-961 (1998).

46. Fink, G. R. \& Guthrie, C. Guide to yeast genetics and molecular biology. (1991).

47. Sikorski, R. S. \& Hieter, P. A system of shuttle vectors and yeast host strains designed for efficient manipulation of DNA in Saccharomyces cerevisiae. Genetics 122, 19-27 (1989).

48. Christianson, T. W., Sikorski, R. S., Dante, M., Shero, J. H. \& Hieter, P. Multifunctional yeast high-copy-number shuttle vectors. Gene 110, 119-122 (1992).

49. Keefe, A. D., Wilson, D. S., Seelig, B. \& Szostak, J. W. One-step purification of recombinant proteins using a nanomolar-affinity streptavidin-binding peptide, the SBP-Tag. Protein Expr. Purif. 23, 440-446 (2001).

50. Natarajan, N., Foresti, O., Wendrich, K., Stein, A. \& Carvalho, P. Quality control of protein complex assembly by a transmembrane recognition factor. Mol. Cell 77, 108-119 (2020). e9.

51. Caesar, J. et al. SIMPLE 3.0. Stream single-particle cryo-EM analysis in real time. J. Struct. Biol.: X 4, 100040 (2020).

52. Zivanov, J. et al. New tools for automated high-resolution cryo-EM structure determination in RELION-3. Elife 7, (2018).

53. Brown, A. et al. Tools for macromolecular model building and refinement into electron cryo-microscopy reconstructions. Acta Crystallogr. Sect. D., Biol. Crystallogr. 71, 136-153 (2015)

54. Afonine, P. V. et al. Real-space refinement in PHENIX for cryo-EM and crystallography. Acta Crystallogr. D. Struct. Biol. 74, 531-544 (2018).

55. Prisant, M. G., Williams, C. J., Chen, V. B., Richardson, J. S. \& Richardson, D. C. New tools in MolProbity validation: CaBLAM for CryoEM backbone, UnDowser to rethink "waters," and NGL viewer to recapture online 3D graphics. Protein Sci. 29, 315-329 (2020).

56. Winn, M. D. et al. Overview of the CCP4 suite and current developments. Acta Crystallogr. Sect. D., Biol. Crystallogr. 67, 235-242 (2011).

57. Krissinel, E. \& Henrick, K. Secondary-structure matching (SSM), a new tool for fast protein structure alignment in three dimensions. Acta Crystallogr. Sect. D., Biol. Crystallogr. 60, 2256-2268 (2004).

58. Marrink, S. J., Risselada, H. J., Yefimov, S., Tieleman, D. P. \& de Vries, A. H. The MARTINI force field: coarse grained model for biomolecular simulations. J. Phys. Chem. B 111, 7812-7824 (2007).

59. Monticelli, L. et al. The MARTINI coarse-grained force field: extension to proteins. J. Chem. Theory Comput. 4, 819-834 (2008).

60. Waterhouse, A. et al. SWISS-MODEL: homology modelling of protein structures and complexes. Nucleic Acids Res. 46, W296-W303 (2018).

61. PLUMED consortium. Promoting transparency and reproducibility in enhanced molecular simulations. Nat. Methods 16, 670-673 (2019).

62. Wassenaar, T. A., Ingólfsson, H. I., Böckmann, R. A., Tieleman, D. P. \& Marrink, S. J. Computational Lipidomics with insane: a versatile tool for generating custom membranes for molecular simulations. J. Chem. Theory Comput. 11, 2144-2155 (2015).

63. Bussi, G., Donadio, D. \& Parrinello, M. Canonical sampling through velocity rescaling. J. Chem. Phys. 126, 014101 (2007)

64. Parrinello, M. Polymorphic transitions in single crystals: a new molecular dynamics method. J. Appl. Phys. 52, 7182 (1981).

65. Vickery, O. N. \& Stansfeld, P. J. CG2AT2: an enhanced fragment-based approach for serial multi-scale molecular dynamics simulations. BioRxiv (2021). https://doi.org/10.1101/2021.03.25.437005.

66. Berendsen, H. J. C., van der Spoel, D. \& van Drunen, R. GROMACS: a message-passing parallel molecular dynamics implementation. Comput Phys. Commun. 91, 43-56 (1995). 
67. Hunter, J. D. Matplotlib: A 2D graphics environment. IEEE Annals of the History of Computing (2007).

68. Humphrey, W., Dalke, A. \& Schulten, K. VMD: visual molecular dynamics. J. Mol. Graph 14, 33-38 (1996). 27.

69. Carvalho, P., Stanley, A. M. \& Rapoport, T. A. Retrotranslocation of a misfolded luminal ER protein by the ubiquitin-ligase Hrdlp. Cell 143, 579-591 (2010).

70. Kushnirov, V. V. Rapid and reliable protein extraction from yeast. Yeast 16, 857-860 (2000).

71. Laemmli, U. K. Cleavage of structural proteins during the assembly of the head of bacteriophage T4. Nature 227, 680-685 (1970).

\section{Acknowledgements}

We thank R. Klemm for critical reading of the manuscript and J. Ferreira for discussions. P.C. was supported by a BBSRC grant (BB/R018375/1) and an investigator award from Wellcome (202642/Z/16/Z). All electron microscopy data collection and processing were performed at the Central Oxford Structural Microscopy and Imaging Centre (COSMIC), which is supported by the Wellcome Trust (201536), the EPA Cephalosporin Trust, and a Royal Society/Wolfson Foundation Laboratory Refurbishment Grant (WL160052). S.M.L.'s lab was supported by a Wellcome Trust award (219477/Z/19/Z). R.A.C. and P.J.S. are supported by Wellcome (208361/Z/17/Z). P.J.S.'s lab is supported by awards from the BBSRC (BB/P01948X/1, BB/R002517/1, and BB/S003339/1) and MRC (MR/ S009213/1). Simulations were performed using the ARCHER/ARCHER2 UK National Supercomputing Service (http://www.archer.ac.uk) and JADE, provided by HECBioSim, the UK High End Computing Consortium for Biomolecular Simulation (hecbiosim.ac.uk), which is supported by the EPSRC (EP/L000253/1). P.J.S. acknowledges the University of Warwick Scientific Computing Research Technology Platform for computational access.

\section{Author contributions}

Y.A.K. carried out all the biochemical work and together with M.F.R. carried out light microscopy acquisition and data analysis. J.C.D. prepared the cryo-EM grids, collected and processed the EM data and determined the structure guided by S.M.L. R.A.C. performed the molecular dynamics simulations guided by P.J.S. P.C. conceived and guided the project and together with Y.A.K. and M.F.R. wrote the initial draft of the manuscript. All authors commented on drafts of the manuscript.

\section{Competing interests}

The authors declare no competing interests

\section{Additional information}

Supplementary information The online version contains supplementary material available at https://doi.org/10.1038/s41467-021-26162-6.

Correspondence and requests for materials should be addressed to Susan M. Lea or Pedro Carvalho.

Peer review information Nature Communications thanks the anonymous reviewers for their contributions to the peer review of this work. Peer review reports are available.

Reprints and permission information is available at http://www.nature.com/reprints

Publisher's note Springer Nature remains neutral with regard to jurisdictional claims in published maps and institutional affiliations.

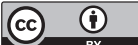

Open Access This article is licensed under a Creative Commons Attribution 4.0 International License, which permits use, sharing, adaptation, distribution and reproduction in any medium or format, as long as you give appropriate credit to the original author(s) and the source, provide a link to the Creative Commons license, and indicate if changes were made. The images or other third party material in this article are included in the article's Creative Commons license, unless indicated otherwise in a credit line to the material. If material is not included in the article's Creative Commons license and your intended use is not permitted by statutory regulation or exceeds the permitted use, you will need to obtain permission directly from the copyright holder. To view a copy of this license, visit http://creativecommons.org/ licenses/by/4.0/

(C) The Author(s) 2021 